\title{
PRODUCT FORMULAS FOR STEENROD OPERATIONS
}

\author{
by ZAIQING LI
}

(Received 3rd August 1993)

\begin{abstract}
A star operation is defined and studied for the Steenrod algebra. Numerous product formulas of Steenrod operations are presented.
\end{abstract}

1991 Mathematics subject classification: Primary 55S10, 16W30, 13A50, 20 C20.

\section{Introduction}

Milnor's composition law [7] (Section 2) has long been the most important theorem for the Steenrod algebra. It is often used to calculate the product of two specific Milnor basic operations. But by manipulating matrices in this calculation one can hardly obtain any formula of general pattern. It is our purpose in this paper that we incorporate Milnor's law into a single product formula of global operations using a star operation among sequences of scalar parameters, which is defined from the coproduct formula of the dual Steenrod algebra (Section 3). Properties of this star operation along with their implications on the Steenrod algebra constitute the main part of this paper. A formula which calculates the conjugate of the Steenrod algebra is an immediate application (Section 4). Weighted symmetric polynomials are defined (Section 5), whose combinatoric properties determine the algebra structure of Steenrod operations. The simplest example of these properties implies the Bullet-Macdonald symmetry for the Adem relations (Section 5). The most general example is a relation between the Dickson and Mui invariants from the modular representation theory and iterated products in the Steenrod algebra (Section 8). We also have more product formulas (Section 6) which are used in a study of nilpotence in the Steenrod algebra (Section 7).

This paper is a revision and extension of the results on the Steenrod algebra first presented in my dissertation. I like to thank sincerely my Ph.D. advisor Professor Richard M. Kane for every piece of advice and guidance I received during my Ph.D. candidacy. I would like to thank Professors Kee-Yuen Lam and Denis K. Sjerve for their supervision and precious help in my post-doctoral years at the University of British Columbia. Finally I would like to thank the Izaak Walton Killam Committee of Canada for awarding me a fellowship under which this paper is written and published.

\section{Milnor Composition Law}

In this section we set up our background. Let $\ell \geqq 1$ be a fixed integer, $p$ a prime. Let $q=q^{\ell}, F_{q}$ a field of $q$ elements. Binomial and multinomial coefficients are denoted by 


$$
\left[\begin{array}{c}
m \\
n
\end{array}\right]=\frac{m(m-1) \ldots(m-n+1)}{n !}, \quad\left[k_{1}, k_{2}, \ldots, k_{n}\right]=\frac{\left(k_{1}+k_{2}+\cdots+k_{n}\right) !}{k_{1} ! k_{2} ! \ldots k_{n} !}
$$

It is true that for non-negative integers $x=\sum_{i \geqq 0} x_{i} p^{i}$ and $y=\sum_{i \geqq 0} y_{i} p^{i}$ in $p$-adic expansion,

$$
\left[\begin{array}{l}
x \\
y
\end{array}\right] \equiv \prod_{i \geqq 0}\left[\begin{array}{l}
x_{i} \\
y_{i}
\end{array}\right](\bmod p), \quad[x, y] \equiv \prod_{i \geqq 0}\left[x_{i}, y_{i}\right](\bmod p)
$$

By an exponent sequence we mean a sequence of non-negative integers $R=$ $\left(r_{1}, r_{2}, r_{3}, \ldots\right)$ such that $r_{i} \neq 0$ for only finite number of $i$ 's. An exponent sequence can be multiplied by a non-negative integer and two exponent sequences can be added component-wise. The following is standard:

$$
\begin{gathered}
|R|=r_{1}+p r_{2}+p^{2} r_{3}+\cdots, \\
\|R\|=2(p-1) r_{1}+2\left(p^{2}-1\right) r_{2}+2\left(p^{3}-1\right) r_{3}+\cdots \\
d(R)=r_{1}+r_{2}+r_{3}+\cdots \\
D(R)=r_{1}+(1+p) r_{2}+\left(1+p+p^{2}\right) r_{3}+\cdots, \\
\Delta_{\ell}=(0, \ldots, 0,1,0, \ldots), \quad \text { where } 1 \text { is the } \ell \text { th component, } \\
0=(0,0,0, \ldots) .
\end{gathered}
$$

By a sequence of scalar parameters we mean a sequence $\xi=\left(\xi_{1}, \xi_{2}, \xi_{3}, \ldots\right)$ which is independent of the Hopf algebra (the Steenrod algebra) that is in discussion. We write $\xi^{R}$ for the monomial $\xi_{1}^{r_{1}} \xi_{2}^{r_{2}} \xi_{3}^{r^{3}} \ldots$ and assume $\xi_{0}=1$ for convenience. When we expand an expression $A$ in terms of monomials $B$ we denote by

$$
\left[\begin{array}{l}
A \\
B
\end{array}\right]
$$

the coefficient of $B$. For example, if $m=k_{1}+k_{2}+\cdots+k_{n}$,

$$
\left[\begin{array}{c}
\left(\xi_{1}+\xi_{2}+\cdots+\xi_{n}\right)^{m} \\
\xi_{1}^{k_{1}} \xi_{2}^{k_{2}} \cdots \xi_{n}^{k_{n}}
\end{array}\right]=\left[k_{1}, k_{2}, \ldots, k_{n}\right]
$$

$\mathscr{A}(p)$ is the Steenrod algebra of $\bmod p$ stable cohomology operations. It is the graded associative algebra over $\mathbb{F}_{p}$ generated by the Bockstein operation $\delta$ and the (reduced) Steenrod operations $\mathscr{P}^{n}(n \geqq 1)$, when $p$ is odd, or by the Steenrod squares $\operatorname{Sq}^{n}(n \geqq 1)$, 
when $p=2$, modulo the Adem relations. Milnor showed that $\mathscr{A}(p)$ has a linear basis containing $\mathscr{P}^{R}$, called Milnor basic elements (operations), where $R$ are exponent sequences, such that $\mathscr{P}^{R}=\mathscr{P}^{r}$ if $R=(r, 0,0, \ldots)$, and that it is a Hopf algebra over $\mathbb{F}_{p}$, the coalgebra structure being the Cartan formula. As conventions, $\mathscr{P} r_{1} \ldots, r_{n}$ is 1 if $n=0$ and $\mathscr{P}^{R}$ is the zero operation if $R$ is not an exponent sequence. In the case $p=2$ we assume $\mathscr{P}^{R}$ is the operation $\mathrm{Sq}^{R}$. Moreover

\section{Notation 2.1.}

(1) $\mathscr{P}_{\ell}^{r}=\mathscr{P}^{r \cdot \Delta \ell}, \quad \mathscr{P}_{\ell}^{t_{1}, t_{2} \ldots, t_{n}}=\mathscr{P}^{t_{1} \Delta \ell+t_{2} \Delta_{2} \ell+\cdots+t_{n} \Delta_{n} \ell}, \quad \mathscr{P}_{\ell}(\alpha)=\sum, \mathscr{P}_{\ell}^{r} \alpha^{r}$

(2) $\mathscr{P}(\xi)=\sum_{R} \mathscr{P}^{R} \xi^{R}, \quad \mathscr{Q}(\eta)=\sum_{k \geqq 0} \mathscr{Q}_{k} \eta_{k}$

To state Milnor's results we define Milnor notations $R(X)=\left(r_{1}, r_{2}, \ldots\right), S(X)=$ $\left(s_{1}, s_{2}, \ldots\right), T(X)=\left(t_{1}, t_{2}, \ldots\right)$ and $b(X)$ for a matrix of non-negative integers, almost all zero,

$$
X=\left(\begin{array}{cccc}
0 & x_{0,1} & x_{0,2} & \cdots \\
x_{1,0} & x_{1,1} & x_{1,2} & \cdots \\
x_{2,0} & x_{2,1} & x_{2,2} & \cdots \\
\vdots & \vdots & \vdots & \ddots
\end{array}\right)
$$

where

$$
r_{i}=\sum_{j=0}^{\infty} p^{j} x_{i, j}, \quad s_{j}=\sum_{i=0}^{\infty} x_{i, j}, \quad t_{k}=\sum_{i+j=k} x_{i, j}, \quad b(X)=\frac{\prod_{k} t_{k} !}{\prod_{i, j} x_{i, j} !}
$$

Theorem $2.2([7])$. The dual Steenrod Algebra $\mathscr{A}_{*}(p)$ is a Hopf algebra over $\mathbb{F}_{p}$. As an algebra, it is Grassmannian:

$$
\mathscr{A}_{*}(p)= \begin{cases}E\left(y_{1}, y_{2}, \ldots\right) \otimes \mathbb{F}_{p}\left[x_{1}, x_{2}, x_{3}, \ldots\right] & \text { if } p \neq 2 \\ \mathbb{F}_{2}\left[x_{1}, x_{2}, x_{3}, \ldots\right] & \text { if } p=2,\end{cases}
$$

where the generators have degree

$$
\operatorname{deg}\left(y_{k}\right)=1, \quad \operatorname{deg}\left(x_{k}\right)= \begin{cases}2\left(p^{k}-1\right) & \text { if } p \neq 2 \\ 2^{k}-1 & \text { if } p=2, \quad k \geqq 1\end{cases}
$$

The coalgebra map $\psi_{*}$ is given by

$$
\begin{array}{ll}
\psi_{*}\left(x_{k}\right)=\sum_{i+j=k} x_{i}^{p^{j}} \otimes x_{j}, & x_{0}=1 \\
\psi_{*}\left(y_{k}\right)=\sum_{i+j=k} x_{i}^{p^{j}} \otimes y_{j}+y_{k} \otimes 1, & y_{0}=1, \quad k \geqq 1 .
\end{array}
$$


Theorem 2.3 (Milnor Composition Law [7]). The Steenrod algebra $\mathscr{A}(p)$ is the Hopf algebra dual to $\mathscr{A}_{*}(p)$. It has a free $\mathbb{F}_{p}$-basis

$$
\begin{array}{ll}
\left\{\mathscr{Q}_{0}^{\varepsilon_{0}} \mathscr{Q}_{1}^{\varepsilon_{1}} \ldots \mathscr{P P}^{R} \mid \varepsilon_{i}=0,1, R: \text { exponent sequences }\right\} & \text { if } p \neq 2, \\
\left\{\mathrm{Sq}^{R} \mid R: \text { exponent sequences }\right\} & \text { if } p=2 .
\end{array}
$$

The degrees are $\operatorname{deg}\left(\mathscr{Q}_{i}\right)=1, \operatorname{deg}\left(\mathscr{P}^{R}\right)=\|R\|$ and $\operatorname{deg}\left(\mathrm{Sq}^{R}\right)=D(R)$. The coalgebra map $\varphi^{*}$ is given by the Cartan formula

$$
\varphi^{*}\left(\mathscr{P}^{R}\right)=\sum_{R_{1}+R_{2}=R} \mathscr{P}^{R_{1}} \otimes \mathscr{P}^{R_{2}}, \quad \varphi^{*}\left(\mathscr{Q}_{k}\right)=\mathscr{Q}_{k} \otimes 1+1 \otimes \mathscr{Q}_{k}
$$

The algebra map $\psi^{*}$ is determined by

(1) $\mathscr{P}^{R} \mathscr{P}^{S}=\sum_{R(X)=R, S(X)=S} b(X) \mathscr{P}^{T(X)}$,

(2) $\mathscr{P}^{R} \mathscr{Q}_{k}=\mathscr{Q}_{k} \mathscr{P}^{R}+\mathscr{Q}_{k+1} \mathscr{P}^{R-p^{k} \Delta_{1}}+\mathscr{Q}_{k+2} \mathscr{P}^{R-p^{k} \Delta_{2}}+\cdots$

(3) $\mathscr{Q}_{i}^{2}=0, \quad \mathscr{Q}_{i} \mathscr{Q}_{j}+\mathscr{Q}_{j} \mathscr{Q}_{i}=0, \quad i \neq j$.

\section{Star operation}

We are going to define a star operation for the Steenrod algebra $\mathscr{A}(p)$. We begin with a Hopf algebra $\left(H_{*}, \varphi_{*}, \psi_{*}\right)$ of finite type over a (graded-)commutative ring $\mathbb{F}$ in the sense of Milnor and Moore [8]. We assume that $H_{*}$ is evenly-graded if the characteristic of $\mathbb{F}$ is not 2 , for this eliminates any sign change when switching order of factors in a product. Suppose that the algebra structure is polynomial $H_{*}=$ $\mathbb{F}\left[x_{1}, x_{2}, \ldots, x_{n}, \ldots\right]$. Hence the dual Hopf algebra $\left(H^{*}, \psi^{*}, \varphi^{*}\right)$ has a free $\mathbb{F}$-basis $\left\{\mathscr{D}^{*} \mid R\right.$ exponent sequences\}:

$$
\left\langle\mathscr{D}^{R} \mid x^{S}\right\rangle= \begin{cases}1 & \text { if } R=S \\ 0 & \text { otherwise }\end{cases}
$$

where $\langle\mid\rangle$ denotes the Kronecker pairing. By duality

$$
\left\langle\varphi^{*}(u) \mid f \otimes g\right\rangle=\left\langle u \mid \varphi_{*}(f \otimes g)\right\rangle,\left\langle\psi^{*}(u \otimes v\rangle \mid f\right\rangle=\left\langle u \otimes v \mid \psi_{*}(f)\right\rangle .
$$

The coalgebra structure $\varphi^{*}$ satisfies the Cartan formula

$$
\varphi^{*}\left(\mathscr{D}^{R}\right)=\sum_{R_{1}+R_{2}=R} \mathscr{D}^{R_{1}} \otimes \mathscr{D}^{R_{2}}
$$

Definition 3.1. Suppose that $\xi=\left(\xi_{1}, \xi_{2}, \ldots\right)$ and $\eta=\left(\eta_{1}, \eta_{2}, \ldots\right)$ are sequences of scalar parameters, and that 


$$
\psi_{*}\left(x_{k}\right)=\sum_{R, S} a_{R, S}^{(k)} x^{R} \otimes x^{S}, \quad a_{R, S}^{(k)} \in \mathbb{F}, \quad k \geqq 1
$$

Then the star operation is $\xi * \eta=\left((\xi * \eta)_{1},(\xi * \eta)_{2}, \ldots\right)$ where

$$
(\xi * \eta)_{k}=\sum_{R, S} a_{R, s}^{(k)} \xi^{R} \eta^{s}, \quad k \geqq 1
$$

Theorem 3.2. For any sequences of scalar parameters $\xi$ and $\eta$,

$$
\sum_{R, S} \mathscr{D}^{R} \mathscr{D}^{S} \xi^{R} \eta^{S}=\sum_{T} \mathscr{D}^{T}(\xi * \eta)^{T}
$$

This means that the product $\mathscr{D}^{R} \mathscr{D}^{S}$ is a sum of $\mathscr{D}^{T}$ with coefficients

$$
\left[\begin{array}{c}
(\xi * \eta)^{T} \\
\xi^{R} \eta^{S}
\end{array}\right] \in \mathbb{F}
$$

Theorem 3.2 will be applied to the Steenrod algebra with a little modification. We then obtain many new and old formulas which constitute the main part of this paper. In [6] a star operation for the Brown-Peterson algebra BP*(BP) is defined. In that case the product formula (3.2) implies the Quillen theorem. We now prove the theorem by an argument of duality.

Lemma 3.3. Let $\mathscr{D}(\zeta)=\sum_{R} \mathscr{D}^{R} \xi^{R}$, and $\mathscr{D}(\eta)=\sum_{s} \mathscr{D}^{s} \eta^{s}$. Then for $f, g \in H_{*}$,

$$
\langle\mathscr{D}(\xi) \cdot \mathscr{D}(\eta) \mid f \cdot g\rangle=\langle\mathscr{D}(\xi) \cdot \mathscr{D}(\eta) \mid f\rangle \cdot\langle\mathscr{D}(\xi) \cdot \mathscr{D}(\eta) \mid g\rangle .
$$

Proof. Since $H^{*}$ is a Hopf algebra $\varphi^{*}$ is multiplicative. By duality and the Cartan formula,

$$
\begin{aligned}
& \left\langle\sum_{R, S} \mathscr{D}^{R} \mathscr{D}^{S} \xi^{R} \eta^{s} \mid f \cdot g\right\rangle \\
& =\left\langle\sum_{R, S} \varphi^{*}\left(\mathscr{D}^{R} \mathscr{D}^{S}\right) \xi^{R} \eta^{s} \mid f \otimes g\right\rangle \\
& =\left\langle\sum_{R, S} \varphi^{*}\left(\mathscr{D}^{R}\right) \varphi^{*}\left(\mathscr{D}^{S}\right) \xi^{R} \eta^{s} \mid f \otimes g\right\rangle \\
& =\sum_{R, S}\left(\sum_{R_{1}+R_{2}=R, s_{1}+S_{2}=S}\left\langle\left(\mathscr{D}^{R_{1}} \otimes \mathscr{D}^{R_{2}}\right) \cdot\left(\mathscr{D}^{s_{1}} \otimes D^{S_{2}}\right) \mid f \otimes g\right\rangle\right) \xi^{R} \cdot \eta^{S} \\
& =\left\langle\sum_{R_{1}, S_{1}} \mathscr{D}^{R_{1}} \mathscr{D}^{S_{1}} \xi^{R_{1}} \eta^{s_{1}} \mid f\right\rangle \cdot\left\langle\sum_{R_{2,} S_{2}} \mathscr{D}^{R_{2}} \mathscr{D}^{S_{2}} \xi^{R_{2}} \eta^{S_{2}} \mid g\right\rangle
\end{aligned}
$$




$$
=\left\langle\sum_{R, S} \mathscr{D}^{R} \mathscr{D}^{S} \xi^{R} \eta^{s} \mid f\right\rangle \cdot\left\langle\sum_{R, S} \mathscr{D}^{R} \mathscr{D}^{S} \xi^{R} \eta^{s} \mid g\right\rangle .
$$

Proof of Theorem 3.2. We check the two sides of the formula in the theorem have the same effect on $H_{*}$ with respect to the Kronecker pairing. For exponent sequence $T$

$$
\begin{aligned}
\left\langle\sum_{R, S} \mathscr{D}^{R} \mathscr{D}^{S} \xi^{R} \eta^{S} \mid x^{T}\right\rangle & =\prod_{k=1}^{\infty}\left\langle\sum_{R, S} D^{R} D^{S} \xi^{R} \eta^{s} \mid x_{k}\right\rangle^{t_{k}} \text { by Lemma } 3.3 \\
& =\prod_{k=1}^{\infty}\left\langle\sum_{R, S} \psi^{*}\left(\mathscr{D}^{R} \otimes D^{S}\right) \xi^{R} \eta^{S} \mid x_{k}\right\rangle^{t_{k}} \\
& =\prod_{k=1}^{\infty}\left(\sum_{R, S}\left\langle\mathscr{D}^{R} \otimes D^{S} \mid \psi^{*}\left(x_{k}\right)\right\rangle \xi^{R} \eta^{S}\right)^{t_{k}} \\
& =\prod_{k=1}^{\infty}\left(\sum_{R, S}\left\langle\mathscr{D}^{R} \otimes D^{s} \mid \sum_{U, V} a_{U, V}^{(k)} x^{U} \otimes x^{V}\right\rangle \xi^{R} \eta^{S}\right)^{t_{k}} \\
& =\prod_{k=1}^{\infty}\left(\sum_{R, S} a_{R, s}^{(k)} \xi^{R} \eta^{s}\right)^{t_{k}} \\
& =(\xi * \eta)^{T} \\
& =\left\langle\sum_{U} \mathscr{D}^{U}(\xi * \eta)^{U} \mid x^{T}\right\rangle
\end{aligned}
$$

We now turn to the case of the Steenrod algebra $\mathscr{A}(p)$. Consider the Hopf algebra $\mathscr{B}_{*}(p)$ which is dual to

$$
\mathscr{B} *(p)= \begin{cases}\mathscr{A}(p) /(\delta) & p \neq 2 \\ \mathscr{A}(2) & p=2\end{cases}
$$

By Theorems 2.2 and $2.3 \mathscr{B}_{*}(p)$ is polynomial: $\mathscr{B}_{*}(p)=\mathbb{F}_{p}\left[x_{1}, x_{2}, x_{3}, \ldots\right]$. The coalgebra structure $\psi_{*}$ is given by

$$
\psi_{*}\left(x_{k}\right)=\sum_{i+j=k} x_{i}^{p^{j}} \otimes x_{j}, \quad k \geqq 1
$$

The dual of $x^{R}$ gives the Milnor basic operation $\mathscr{P}^{R}$. Therefore a star operation is defined for $\mathscr{B}_{*}(p)$ hence for the Steenrod algebra $\mathscr{A}(p)$. For sequences of scalar parameters $\xi$ and $\eta$, we define $\xi * \eta$ by

$$
(\xi * \eta)_{k}=\sum_{i+j=k} \xi_{i}^{j} \eta_{j}, \quad k \geqq 1
$$


Remark 3.4. The polynomials $(\xi * \eta)_{k}$ are in the first column of the product of matrices $\bmod p$ :

$$
\left(\begin{array}{ccccc}
1 & 0 & 0 & 0 & \ldots \\
\xi_{1} & 1 & 0 & 0 & \ldots \\
\xi_{2} & \xi_{1}^{p} & 1 & 0 & \ldots \\
\xi_{3} & \xi_{2}^{p} & \xi_{1}^{p^{2}} & 1 & \ldots \\
\vdots & \vdots & \vdots & \vdots & \ddots
\end{array}\right) \cdot\left(\begin{array}{ccccc}
1 & 0 & 0 & 0 & \ldots \\
\eta_{1} & 1 & 0 & 0 & \ldots \\
\eta_{2} & \eta_{1}^{p} & 1 & 0 & \ldots \\
\eta_{3} & \eta_{2}^{p} & \eta_{1}^{\mathrm{p}^{2}} & 1 & \ldots \\
\vdots & \vdots & \vdots & \vdots & \ddots
\end{array}\right) .
$$

It is true that for any $\xi, \eta$ and $\zeta$, there is an $\bar{\xi}$ such that

$$
(\xi * \eta) * \zeta=\xi *(\eta * \zeta), \quad \xi * 0=\xi=0 * \xi, \quad \xi * \bar{\zeta}=0=\bar{\xi} * \xi .
$$

Theorem 3.5 (Milnor Product Formula).

(1) $\mathscr{P}(\xi) \cdot \mathscr{P}(\eta)=\mathscr{P}(\xi * \eta)$,

(2) $\mathscr{P}(\xi) \cdot \mathscr{Q}(\eta)=\mathscr{Q}(\xi * \eta) \cdot \mathscr{P}(\xi)$,

(3) $2(\xi) \cdot \mathscr{Q}(\eta)+\mathscr{Q}(\eta) \cdot \mathscr{Q}(\xi)=0$.

Proof. (1) is a direct application of Theorem 3.2 when applied to the Hopf algebra $\mathscr{B}^{*}(p)$. (2) and (3) are reformulations of Theorem 2.3(2) and (3) using global operations.

Global operations $\mathscr{P}(\xi)$ are parallel with those in Atiyah and Hirzebruch [1] and Monks [9]. We use the star operation in the proof of a theorem [9] which is credited by Monks as having originally been proved by Atiyah and Hirzebuch.

Theorem $3.6([1])$. Let $S\left(\varepsilon_{1}, \varepsilon_{2}, \ldots\right)=\sum_{r_{1}, r_{2}, \ldots} \mathrm{Sq}^{\varepsilon_{1} r_{1}, \varepsilon_{2} r_{2}, \ldots,}, \varepsilon_{i}=0,1$, called generalized total Steenrod squares. The set of operations $S\left(\varepsilon_{1}, \varepsilon_{2}, \ldots\right)$ is a group under the multiplication of Steenrod operations. Moreover it is isomorphic to the group of units of the algebras $\mathbb{F}_{2}[[x]]$ of formal power series by the map

$$
S\left(\varepsilon_{1}, \varepsilon_{2}, \ldots\right) \mapsto 1+\varepsilon_{1} x+\varepsilon_{2} x^{2}+\cdots .
$$

Proof. The global operation $S\left(\varepsilon_{1}, \varepsilon_{2}, \ldots\right)$ is equal to $\mathscr{P}(\varepsilon)$ where $\varepsilon=\left(\varepsilon_{1}, \varepsilon_{2}, \ldots\right)$, for

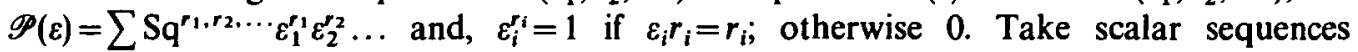
$\lambda=\left(\lambda_{1}, \lambda_{2}, \ldots\right)$ and $\mu=\left(\mu_{1}, \mu_{2} \ldots\right)$ from $\mathbb{F}_{2}$. By Theorem 3.2

$$
S\left(\lambda_{1}, \lambda_{2}, \ldots\right) \cdot S\left(\mu_{1}, \mu_{2}, \ldots\right)=S(\lambda * \mu), \quad(\lambda * \mu)_{k}=\sum_{i+j=k} \lambda_{i}^{j} \mu_{j}=\sum_{i+j=k} \lambda_{i} \mu_{j}
$$

This implies that the multiplication of generalized total Steenrod squares corresponds to 
the multiplication of formal power series from $\mathbb{F}_{2}[[x]]$. It is obvious that the map defined in the theorem is injective and surjective. The theorem follows.

\section{Conjugate}

Our first application of Theorem $3.5(1)$ is to calculate $\chi$ (or the anti-automorphism in [7]) of the Steenrod algebra $\mathscr{A}(p)$ which is defined inductively by

$$
\sum_{R_{1}+R_{2}=R} \chi\left(\mathscr{P}^{R_{1}}\right) \cdot \mathscr{P}^{R_{2}}=\left\{\begin{array}{ll}
0 & \text { if } R \neq 0 \\
1 & \text { if } R=0,
\end{array} \text { or }\left(\sum_{R} \chi\left(\mathscr{P}^{R}\right) \xi^{R}\right) \cdot\left(\sum_{R} \mathscr{P}^{R} \xi^{R}\right)=1\right.
$$

Milnor attempted to calculate $\chi$ non-inductively ([7, Theorem 5]) but finished up with a very complicated formula which is almost impossible to use in practice. Our formula is much simpler.

Theorem 4.1. For any exponent sequence $R$

$$
\chi\left(\mathscr{P}^{R}\right)=(-1)^{d(R)} \cdot \sum_{R(X)=R} b(X) \cdot \mathscr{P}^{T(X)-S(X)}
$$

where $X$ runs over all matrices of non-negative integers, almost all zero, of the form

$$
X=\left(\begin{array}{cccc}
0 & 0 & 0 & \ldots \\
x_{1,0} & x_{1,1} & x_{1,2} & \ldots \\
x_{2,0} & x_{2,1} & x_{2,2} & \ldots \\
\vdots & \vdots & \vdots & \ddots
\end{array}\right)
$$

For example,

$$
\begin{gathered}
\chi\left(\mathscr{P}^{r}\right)=(-1)^{r} \sum_{D(R)=r} \mathscr{P}^{R}, \quad([7]) \\
\chi\left(\mathscr{P}^{R}\right)=(-1)^{d(R)} \mathscr{P}^{R}, \quad \text { if all } r_{i}<p \\
\chi\left(\mathscr{P}^{p \Delta_{n}}\right)=-\mathscr{P}^{p \Delta_{n}}-\mathscr{P}^{\Delta_{n+1}}, \\
\chi\left(\mathrm{Sq}^{3,2,1}\right)=\mathrm{Sq}^{3,2,1}+\mathrm{Sq}^{0,3,1}
\end{gathered}
$$

Proof. Given $\xi$, choose $\eta$ such that $\xi * \eta=0$, i.e. $-\eta_{n}=\xi_{n}+\xi_{n-1}^{p} \eta_{1}+\cdots+\xi_{1}^{p^{n-1}}$ for $n \geqq 1$. By Theorem 3.5(1)

$$
\left(\sum_{R} \mathscr{P}^{R} \xi^{R}\right) \cdot\left(\sum_{S} \mathscr{P}^{S} \eta^{S}\right)=\sum_{T} \mathscr{P}^{T}(\xi * \eta)^{T}=1
$$


Multiplying with $\sum_{R} \chi\left(\mathscr{P}^{R}\right) \xi^{R}$ from the left,

$$
\sum_{S} \mathscr{P}^{S} \eta^{S}=\sum_{R} \chi\left(\mathscr{P}^{R}\right) \xi^{R}, \quad \text { hence } \quad \chi\left(\mathscr{P}^{R}\right)=\sum_{S}\left[\begin{array}{l}
\eta^{S} \\
\xi^{R}
\end{array}\right] \cdot \mathscr{P}^{S}
$$

We claim that for $S=\left(s_{1}, \ldots, s_{n}, 0, \ldots\right), s_{n} \neq 0$,

$$
\eta^{S}=\sum_{T(X)=S+S(X)}(-1)^{d(R(X))} b(X) \cdot \xi^{R(X)}
$$

where

$$
X=\left(\begin{array}{cccccc}
0 & 0 & 0 & \ldots & 0 & 0 \\
x_{1,0} & x_{1,1} & x_{1,2} & \ldots & x_{1, n-1} & 0 \\
x_{2,0} & x_{2,1} & x_{2,2} & \ldots & 0 & 0 \\
\vdots & \vdots & \vdots & \vdots & \vdots & \vdots \\
x_{n-1,0} & x_{n-1,1} & 0 & \ldots & 0 & 0 \\
x_{n, 0} & 0 & 0 & \ldots & 0 & 0
\end{array}\right)
$$

with $x_{i, j}$ non-negative integers. This can be proved by a technique of successive expansion. We ignore the minus sign of $\eta_{n}$ (which can be easily recovered). For $S=\left(s_{1}, \ldots, s_{n}, 0, \ldots\right), s_{n} \neq 0$,

$$
\begin{aligned}
\eta_{n}^{s_{n}}=\sum_{x_{n, 0}+\cdots+x_{1, n-1}=s_{n}}\left[x_{n, 0}, x_{n-1,1}, \ldots, x_{1, n-1}\right] \cdot \xi_{1}^{p^{n-1} x_{1, n-1}} \ldots \xi_{n-1}^{p x_{n}-1,1} \xi_{n}^{x_{n, 0}} \cdot \eta_{1}^{x_{n-1,1}} \ldots \\
\eta_{n-2,2}^{x_{2, n} \eta_{n-1}^{x_{1}, n-1} .}
\end{aligned}
$$

Let $S^{\prime}=\left(s_{1}+x_{n-1,1}, s_{2}+x_{2, n-2}, \ldots, s_{n-1}+x_{1, n-1}, 0, \ldots\right)$. Now by induction on $n$

$$
\eta^{S^{\prime}}=\sum_{T(Y)=S^{\prime}+S(Y)} b(Y) \cdot \xi^{R(Y)}
$$

where

$$
X=\left(\begin{array}{cccccc}
0 & 0 & 0 & \ldots & 0 & 0 \\
x_{1,0} & x_{1,1} & x_{1,2} & \ldots & x_{1, n-2} & 0 \\
x_{2,0} & x_{2,1} & x_{2,2} & \ldots & 0 & 0 \\
\vdots & \vdots & \vdots & \vdots & \vdots & \vdots \\
x_{n-2,0} & x_{n-2,1} & 0 & \ldots & 0 & 0 \\
x_{n-1,0} & 0 & 0 & \ldots & 0 & 0
\end{array}\right)
$$

with $x_{i, j}$ non-negative integers. We find that $Y$ is the upper-left $(n \times n)$-corner in the matrix $X$, except the integers $x_{i, j}, i+j=n$. Therefore 


$$
\begin{aligned}
\eta^{S} & =\left(\eta_{1}^{s_{1}} \ldots \eta_{n-1}^{s_{n-1}}\right) \cdot \eta_{n}^{s_{n}} \\
& =\sum_{x_{n, 0}+\cdots+x_{1, n-1}=s_{n}}\left[x_{n, 0}, x_{n-1,1}, \ldots, x_{1, n-1}\right] \cdot \xi_{1}^{p^{n-1} x_{1, n-1}} \ldots \xi_{n-1}^{p x_{n-1,1}} \xi_{n}^{x_{n, 0} \cdot \eta^{s^{\prime}}} \\
& =\sum_{x_{n, 0}+\cdots+x_{1, n-1}=s_{n}, T(Y)=s^{\prime}+S(Y)}\left[x_{n, 0}, x_{n-1,1}, \ldots, x_{1, n-1}\right] \cdot \xi_{1}^{p^{n-1} x_{1, n-1}} \ldots \xi_{n-1}^{p x_{n}-1,1} \xi_{n}^{x_{n, 0}} \cdot \xi^{R(Y)} \\
& =\sum_{T(X)=S+S(X)} b(X) \cdot \xi^{R(X)} .
\end{aligned}
$$

This proves the claim. We are done by reading off from it the coefficient $\left[\begin{array}{l}\eta^{s} \\ \xi^{R}\end{array}\right]$.

Formulas involving the conjugate $\chi$ can also be given in the form of global operations. The following is such an example.

Theorem 4.2. For scalar parameters $\alpha$ and $\beta$,

(1) $\sum_{r, m}(-1)^{m} \mathscr{P}^{r} \chi\left(\mathscr{P}^{m}\right) \alpha^{r} \beta^{m}=\sum_{T} \mathscr{P}^{T}(\alpha+\beta)^{|T|} \beta^{D\left(T^{*}\right)}$

(2) $\sum_{m, r}(-1)^{m} \chi\left(\mathscr{P}^{m}\right) \mathscr{P}^{r} \beta^{m} \alpha^{r}=\sum_{T} \mathscr{P P}^{T}(\alpha+\beta)^{d(T)} \beta^{p D\left(T^{\prime}\right)}$.

In other words,

$$
\begin{aligned}
& \mathscr{P P}^{r} \chi\left(\mathscr{P}^{m}\right)=(-1)^{m} \sum_{D(T)=m+r}\left[\begin{array}{c}
|T| \\
r
\end{array}\right] \mathscr{P} r \\
& \chi\left(\mathscr{P}^{m}\right) \mathscr{P}^{r}=(-1)^{m} \sum_{D(T)=m+r}\left[\begin{array}{c}
d(T) \\
r
\end{array}\right] \mathscr{P} r
\end{aligned}
$$

Proof. Take $\xi=(\alpha, 0,0, \ldots), \eta=(-\beta, 0,0, \ldots)$ and $\zeta=\left(\beta, \beta^{p+1}, \beta^{p^{2}+p+1}, \ldots\right)$. Then $\eta * \zeta=0$ and $\xi * \zeta=\left(\alpha+\beta,(\alpha+\beta)^{p} \beta,(\alpha+\beta)^{p^{2}} \beta^{p+1}, \ldots\right)$. By Theorem 3.5

$$
\sum_{R, S} \mathscr{P P}^{R} \mathscr{P}^{S} \xi^{R} \zeta^{S}=\sum_{T} \mathscr{P}^{T}(\xi * \zeta)^{T} \quad \sum_{U, S} \mathscr{P}^{U} \mathscr{P}^{S} \eta^{U} \zeta^{S}=\sum_{T} \mathscr{P}^{T}(\eta * \zeta)^{T}
$$

That is,

$$
\sum_{r, S} \mathscr{P}^{r} \mathscr{P}^{S} \alpha^{r} \zeta^{S}=\sum_{T} \mathscr{P}^{T}(\alpha+\beta)^{|T|} \beta^{D\left(T^{\prime}\right)}, \quad \sum_{U, S} \mathscr{P}^{U} \mathscr{P P}^{S} \eta^{U} \zeta^{S}=1
$$

The latter identity implies

$$
\sum_{S} \mathscr{P}^{s} \zeta^{s}=\sum_{U} \chi\left(\mathscr{P}^{U}\right) \eta^{U}=\sum_{m}(-1)^{m} \chi\left(\mathscr{P}^{m}\right) \beta^{m},
$$

which is substituted into the left hand side of the former identity. We get the first formula in the theorem. The second one can be treated similarly. 


\section{Iterated products}

Definition 5.1. Suppose $\alpha_{1}, \alpha_{2}, \ldots, \alpha_{n}$ are scalar parameters. The $q$-weighted symmetric polynomials for the $\alpha_{i}$ are

$$
\left\{\begin{array}{l}
\sigma_{1}=\alpha_{1}+\alpha_{2}+\cdots+\alpha_{n} \\
\sigma_{2}=\alpha_{1}^{q} \alpha_{2}+\cdots+\alpha_{1}^{q} \alpha_{n}+\alpha_{2}^{q} \alpha_{3}+\cdots+\alpha_{n-1}^{q} \alpha_{n} \\
\sigma_{3}=\alpha_{1}^{q^{2}} \alpha_{2}^{q} \alpha_{3}+\alpha_{1}^{q^{2}} \alpha_{2}^{q} \alpha_{4}+\cdots+\alpha_{n-2}^{q^{2}} \alpha_{n-1}^{q} \alpha_{n} \\
\quad \vdots \\
\sigma_{n}=\alpha_{1}^{q^{n-1}} \alpha_{2}^{q^{n-2}} \cdots \alpha_{n}
\end{array}\right.
$$

Notice that if the elements $\alpha_{1}, \alpha_{2}, \ldots, \alpha_{n}$ are algebraically independent over $\mathbb{F}_{p}$, then so are the elements $\sigma_{1}, \sigma_{2}, \ldots, \sigma_{n}$. In fact $\alpha_{i}$ is algebraic over $\mathbb{F}_{p}\left(\alpha_{i+1}, \ldots, \alpha_{n}, \sigma_{1}, \sigma_{2}, \ldots, \sigma_{n}\right)$ for $n \geqq i \geqq 1$. For example $\sigma_{n}$ satisfies the equation

$$
\alpha_{n}^{\left(q^{n}-1\right) /(q-1)}-\sigma_{1}^{q^{n-1}} \alpha_{n}^{\left(q^{n-1}-1\right) /(q-1)}+\cdots+(-1)^{n-1} \sigma_{n-1}^{q} \alpha_{n}^{(q-1) /(q-1)}+(-1)^{n} \sigma_{n}=0 .
$$

Theorem 5.2. Let $\alpha_{1}, \alpha_{2}, \ldots, \alpha_{n}$ and $\sigma_{1}, \sigma_{2}, \ldots, \sigma_{n}$ be as in Definition 5.1. Then

$$
\sum_{r_{1}, r_{2}, \ldots, r_{n}} \mathscr{P}_{\ell}^{r_{1}} \mathscr{P P}_{\ell}^{r_{2}} \ldots \mathscr{P}_{\ell}^{r_{n}} \cdot \alpha_{1}^{r_{1}} \alpha_{2}^{r_{2}} \ldots \alpha_{n}^{r_{n}}=\sum_{t_{1}, t_{2}, \ldots, t_{n}} \mathscr{P}_{\ell}^{t_{1}}, \ldots, t_{n} \cdot \sigma_{1}^{t_{1}} \sigma_{2}^{t_{2}} \ldots \sigma_{n}^{t_{n}}
$$

Proof. Since the star operation is associative it follows from the iteration of Theorem 3.5 that for sequences of scalar parameters $\xi_{(k)}, k=1,2, \ldots, n$,

$$
\mathscr{P}\left(\xi_{(1)}\right) \mathscr{P}\left(\xi_{(2)}\right) \ldots \mathscr{P}\left(\xi_{(n)}\right)=\mathscr{P}\left(\xi_{(1)} * \xi_{(2)} * \ldots * \xi_{(n)}\right) .
$$

In this case $\xi_{(i)}=\left(0, \ldots, 0, \alpha_{i}, 0, \ldots\right)$ the sequence $\xi_{(1)} * \xi_{(2)} * \ldots * \xi_{(n)}$ consists of the elements appearing in the first column of a product of $n$ matrices, e.g. in the case $\ell=1$ (by Remark 3.5)

$$
\left(\begin{array}{ccccc}
1 & 0 & 0 & 0 & \ldots \\
\alpha_{1} & 1 & 0 & 0 & \ldots \\
0 & \alpha_{1}^{p} & 1 & 0 & \ldots \\
0 & 0 & \alpha_{1}^{p^{2}} & 1 & \ldots \\
\vdots & \vdots & \vdots & \vdots & \ddots
\end{array}\right)\left(\begin{array}{ccccc}
1 & 0 & 0 & 0 & \ldots \\
\alpha_{2} & 1 & 0 & 0 & \ldots \\
0 & \alpha_{2}^{p} & 1 & 0 & \ldots \\
0 & 0 & \alpha_{2}^{p^{2}} & 1 & \ldots \\
\vdots & \vdots & \vdots & \vdots & \ddots
\end{array}\right) \ldots\left(\begin{array}{ccccc}
1 & 0 & 0 & 0 & \ldots \\
\alpha_{n} & 1 & 0 & 0 & \ldots \\
0 & \alpha_{n}^{p} & 1 & 0 & \ldots \\
0 & 0 & \alpha_{n}^{p^{2}} & 1 & \ldots \\
\vdots & \vdots & \vdots & \vdots & \ddots
\end{array}\right) .
$$

They are the elements of $\left(\sigma_{1}, \sigma_{2}, \ldots, \sigma_{n}, 0, \ldots\right)$. The theorem follows.

We see that iterated products of Steenrod operations are completely determined by the combinatoric behaviors of weighted symmetric polynomials. Of numerous consequences the Peterson formula and the Bullet-Macdonald symmetry for the Adem relations are immediate from Theorem 5.2. 
Theorem 5.3 ([12]). For $m, n \geqq 1$

$$
\sum_{r_{1}+r_{2}+\cdots+r_{n}=m} \mathscr{P}_{\ell}^{r_{1}} \mathscr{P}_{\ell}^{r_{2}} \ldots \mathscr{P}_{\ell}^{r_{n}}=\sum_{t_{1}(q-1)+\cdots+t_{n}\left(q^{n}-1\right)=m(q-1)}\left[\begin{array}{l}
n \\
1
\end{array}\right]^{t_{1}}\left[\begin{array}{l}
n \\
2
\end{array}\right]^{t_{2}} \ldots\left[\begin{array}{l}
n \\
n
\end{array}\right]^{t_{n}} \cdot \mathscr{P}_{\ell}^{t_{1}, t_{2}, \ldots, t_{n}}
$$

In particular when $n$ is a power of $q$,

$$
\sum_{r_{1}+r_{2}+\cdots+r_{n}=m} \mathscr{P}_{\ell}^{r_{1}} \mathscr{P}_{\ell}^{r_{2}} \ldots \mathscr{P}_{\ell}^{r_{n}}= \begin{cases}\mathscr{P}_{\ell}^{0,0}, \ldots, 0, m /\left(1+q+\cdots+q^{n-1}\right) & \text { if }\left(1+q+\cdots+q^{n-1}\right) \mid m \\ 0 & \text { otherwise. }\end{cases}
$$

Proof. The formula 5.2 splits by total degree from the both sides. The identity in degree $2 m(q-1)$ (or in degree $m(q-1)$ if $p=2$ ) is the formula to be proven.

Theorem 5.4. If $\alpha_{1}=u(v-u)^{q-1}$ and $\alpha_{2}=v^{q}$, then

(1) $\mathscr{P}_{\ell}\left(\alpha_{1}\right) \cdot \mathscr{P}_{\ell}\left(\alpha_{2}\right)$, and

(2) $v^{q^{m}}\left(\mathscr{P}_{\ell}\left(\alpha_{1}\right) \cdot \mathscr{Q}_{m \ell}-\mathscr{Q}_{m \ell} \cdot \mathscr{P}_{\ell}\left(\alpha_{1}\right)\right) \cdot \mathscr{P}_{\ell}\left(\alpha_{2}\right), \quad m \geqq 1$

are expressions symmetric with respect to $u$ and $v$.

Proof. (1). A special case of Theorem 3.5(1) is

$$
\mathscr{P}_{\ell}\left(\alpha_{1}\right) \cdot \mathscr{P}_{\ell}\left(\alpha_{2}\right)=\sum_{t_{1,12}} \mathscr{P P}_{\ell}^{t_{1}, t_{2}} \sigma_{1}^{t_{1}} \sigma_{2}^{t_{2}}
$$

where

$$
\left\{\begin{array}{l}
\sigma_{1}=\alpha_{1}+\alpha_{2}=u(v-u)^{q-1}+v^{q}=\frac{u^{q+1}-v^{q+1}}{u-v} \\
\sigma_{2}=\alpha_{1}^{q} \alpha_{2}=(u-v)^{q^{2}-q} u^{q} v^{q}
\end{array}\right.
$$

Since both $\sigma_{1}$ and $\sigma_{2}$ are symmetric with respect to $u$ and $v$, (1) follows.

(2). By Theorem 2.3(2), $\mathscr{P}_{\ell}^{r} \mathscr{Q}_{m \ell}-\mathscr{Q}_{m \ell} \mathscr{P}_{\ell}^{r}=\mathscr{Q}_{(m+1) \ell} \mathscr{P}_{\ell}^{r-q^{m}}$. In terms of global operations,

$$
\mathscr{P}_{\ell}\left(\alpha_{1}\right) \cdot \mathscr{Q}_{m \ell}-\mathscr{Q}_{m \ell} \cdot \mathscr{P}_{\ell}\left(\alpha_{1}\right)=\mathscr{Q}_{(m+1) \ell} \mathscr{P}_{\ell}\left(\alpha_{1}\right) \alpha_{1}^{q^{m}}
$$


Hence (2) is equal to

$$
v^{q^{m}} \mathscr{Q}_{(m+1) \ell} \mathscr{P}_{\ell}\left(\alpha_{1}\right) \alpha_{1}^{q^{m}} \mathscr{P}_{\ell}\left(\alpha_{2}\right)=\mathscr{Q}_{(m+1) \ell}\left(\mathscr{P}_{\ell}\left(\alpha_{1}\right) \cdot \mathscr{P}_{\ell}\left(\alpha_{2}\right)\right)(u v)^{q^{m}}(v-u)^{(q-1) q^{m}}
$$

which is symmetric by (1).

Bullet and Macdonald proved the symmetry above in the case $\ell=1$ and $m=0$ by using a Cartan-Serre theorem. They also showed a technique of residue computation that their symmetry implies the Adem relations. Recall that the Adem relations [13] are

$$
\left\{\begin{aligned}
\mathscr{P}^{a} \mathscr{P}^{b}= & \sum_{j=0}^{[a / p]}(-1)^{a+j}\left[\begin{array}{c}
(p-1)(b-j)-1 \\
a-p j
\end{array}\right] \mathscr{P}^{a+b-j} \mathscr{P}^{j}, \quad 1 \leqq a<p b, \\
\mathscr{P}^{a} \delta \mathscr{P}^{b} & =\sum_{j=0}^{[a / p]}(-1)^{a+j}\left[\begin{array}{c}
(p-1)(b-j)-1 \\
a-p j
\end{array}\right] \delta \mathscr{P}^{a+b-j} \mathscr{P}^{j}, \\
& +\sum_{j=0}^{[(a-1) / p]}(-1)^{a+j-1}\left[\begin{array}{c}
(p-1)(b-j)-1 \\
a-p j-1
\end{array}\right] \mathscr{P}^{a+b-j} \delta \mathscr{P}^{j}, \quad 1 \leqq a<p b, \quad p \neq 2 .
\end{aligned}\right.
$$

They are a set of relations which defines the Steenrod algebra algebraically. The residue technique is applied to Theorem 5.4. Hence the Adem relations for the subalgebra of the Steenrod algebra $\mathscr{A}(p)$ generated by the operations $\mathscr{P}_{l}$ are

$$
\mathscr{P}_{\ell}^{a} \mathscr{P}_{\iota}^{b}=\sum_{j=0}^{[a / q]}(-1)^{a+j}\left[\begin{array}{c}
(q-1)(b-j)-1 \\
a-q j
\end{array}\right] \mathscr{P}_{\ell}^{a+b-j} \mathscr{P}_{\ell}^{j}, \quad a, b \geqq 0 .
$$

Later in Section 7 we will find that there are Adem relations for other algebras as well. In Section 8 we will fully extend the symmetry in Theorem 5.4 to a relation between the Dickson invariants and Mui invariants of modular representation theory.

\section{Product formulas}

Observation 6.1. Let $\xi, \eta$ and $\lambda$ be sequences of scalar parameters. Define a formal power series over $\mathbb{F}_{p}$ by

$$
F=\prod_{k \geqq 1} \frac{1}{1-(\xi * \eta)_{k} \lambda_{k}}
$$

Then Theorem $3.5(1)$ is equivalent to the fact that 


$$
\left[\begin{array}{c}
F \\
\xi^{R} \eta^{S}
\end{array}\right]=\sum_{T} a_{T} \lambda^{T} \Rightarrow \mathscr{P P}^{R} \mathscr{P} S=\sum_{T} a_{T} \mathscr{P}^{T}
$$

For,

$$
\begin{gathered}
F=\prod_{k \geqq 1}\left(\frac{1}{1-(\xi * \eta)_{k} \lambda_{k}}\right)=\sum_{T}(\xi * \eta)^{T} \lambda^{T}, \quad\left[\begin{array}{c}
F \\
\xi^{R} \eta^{S}
\end{array}\right]=\sum_{T}\left[\begin{array}{c}
(\xi * \eta)^{T} \\
\xi^{R} \eta^{S}
\end{array}\right] \lambda^{T} \\
a_{T}=\left[\begin{array}{c}
(\xi * \eta)^{T} \\
\xi^{R} \eta^{S}
\end{array}\right], \quad \mathscr{P}^{R} \mathscr{P}^{S}=\sum_{T}\left[\begin{array}{c}
(\xi * \eta)^{T} \\
\xi^{R} \eta^{S}
\end{array}\right]=\sum_{T} a_{T} \mathscr{P}^{T} .
\end{gathered}
$$

This observation implies:

Rule of thumb. When we have a formula involving coefficients in the expansion of $F$ in terms of $\xi$ and $\eta$, we may convert it into a formula for Steenrod operations by replacing each monomial $\xi^{R} \eta^{S}$ with $\mathscr{P}^{R} \mathscr{P}^{S}$ and $\lambda^{T}$ with $\mathscr{P}^{T}$.

As a simple example let us consider the $\bmod 2$ case where $\xi=(\alpha, 0,0, \ldots)$, $\eta=(\beta, 0,0, \ldots)$ and $\lambda=\left(\lambda_{1}, \lambda_{2}, \ldots\right)$. In other words,

$$
F=\frac{1}{1+(\alpha+\beta) \lambda_{1}} \cdot \frac{1}{1+\alpha^{2} \beta \lambda_{2}}=\sum_{r, s} f_{r, s}\left(\lambda_{1}, \lambda_{2}\right) \alpha^{r} \beta^{s}, \quad \text { over } \mathbb{F}_{2},
$$

where $f_{r, s}\left(\lambda_{1}, \lambda_{2}\right)$ are polynomials in $\lambda_{1}$ and $\lambda_{2}$ over $\mathbb{F}_{2}$. It is true that

$$
\begin{gathered}
F^{2}=\sum_{r, s} f_{r, s}\left(\lambda_{1}^{2}, \lambda_{2}^{2}\right) \alpha^{2 r} \beta^{2 s}, \\
\left(1+(\alpha+\beta) \lambda_{1}\right)\left(1+\alpha^{2} \beta \lambda_{2}\right)=\left(\alpha^{3} \beta \lambda_{1} \lambda_{2}\right)+\left(\alpha \lambda_{1}\right)+\left(\beta \lambda_{1}+\alpha^{2} \beta \lambda_{2}\right)+\left(1+\alpha^{2} \beta^{2} \lambda_{1} \lambda_{2}\right) .
\end{gathered}
$$

Comparing the coefficients of monomials in $\alpha$ and $\beta$ from the both sides of the formula $F=\left(1+(\alpha+\beta) \lambda_{1}\right)\left(1+\alpha^{2} \beta \lambda_{2}\right) \cdot F^{2}$, we find that, for any integers $u$ and $v$

(1) $f_{2 u+1,2 v+1}\left(\lambda_{1}, \lambda_{2}\right)=\lambda_{1} \lambda_{2} f_{u-1, v}\left(\lambda_{1}^{2}, \lambda_{2}^{2}\right)$,

(2) $f_{2 u+1,2 v}\left(\lambda_{1}, \lambda_{2}\right)=\lambda_{1} f_{u, v}\left(\lambda_{1}^{2}, \lambda_{2}^{2}\right)$

(3) $f_{2 u, 2 v+1}\left(\lambda_{1}, \lambda_{2}\right)=\lambda_{1} f_{u, v}\left(\lambda_{1}^{2}, \lambda_{2}^{2}\right)+\lambda_{2} f_{u-1, v}\left(\lambda_{1}^{2}, \lambda_{2}^{2}\right)$,

(4) $f_{2 u, 2 v}\left(\lambda_{1}, \lambda_{2}\right)=f_{u, v}\left(\lambda_{1}^{2}, \lambda_{2}^{2}\right)+\lambda_{1} \lambda_{2} f_{u-1, v}\left(\lambda_{1}^{2}, \lambda_{2}^{2}\right)$.

By our rule of thumb, we obtain, for example, $\mathrm{Sq}^{2 u+1} \mathrm{Sq}^{2 v+1}$ from $f_{2 u+1,2 v+1}$ and $A_{1} A_{2} D\left(\mathrm{Sq}^{u-1} \mathrm{Sq}^{v}\right)$ from $\lambda_{1} \lambda_{2} f_{u-1, v}\left(\lambda_{1}^{2}, \lambda_{2}^{2}\right)$, where $A_{1} A_{2} D$ is the composite of the $\mathbb{F}_{2}$-maps $A_{1}, A_{2}$ and $D$ from $\mathscr{A}(2)$ to itself such that

$$
A_{1}: \mathrm{Sq}^{t_{1}, t_{2}} \mapsto \mathrm{Sq}^{t_{1}+t_{1} t_{2}}, \quad A_{2}: \mathrm{Sq}^{t_{1}, t_{2}} \mapsto \mathrm{Sq}^{t_{1}, t_{2}+1}, \quad D: \mathrm{Sq}^{t_{1}, t_{2}} \mapsto \mathrm{Sq}^{2 t_{1}, 2 t_{2}} .
$$


This proves:

Theorem 6.2. For any integers $u$ and $v$

(1) $\mathrm{Sq}^{2 u+1} \mathrm{Sq}^{2 v+1}=A_{1} A_{2} D\left(\mathrm{Sq}^{u-1} \mathrm{Sq}^{v}\right)$

(2) $\mathrm{Sq}^{2 u+1} \mathrm{Sq}^{2 v}=A_{1} D\left(\mathrm{Sq}^{u} \mathrm{Sq}^{v}\right)$

(3) $\mathrm{Sq}^{2 u} \mathrm{Sq}^{2 v+1}=A_{1} D\left(\mathrm{Sq}^{u} \mathrm{Sq}^{v}\right)+A_{2} D\left(\mathrm{Sq}^{u-1} \mathrm{Sq}^{v}\right)$,

(4) $\mathrm{Sq}^{2 u} \mathrm{Sq}^{2 v}=D\left(\mathrm{Sq}^{u} \mathrm{Sq}^{v}\right)+A_{1} A_{2} D\left(\mathrm{Sq}^{u-1} \mathrm{Sq}^{v-1}\right)$.

We also define $E: \mathrm{Sq}^{t_{1}, t_{2}} \mapsto \mathrm{Sq}^{4 t_{1}+3,4 t_{2}-1}$. It turns out that:

Theorem 6.3. For any integers $u$ and $v$,

$$
\mathrm{Sq}^{4 u} \mathrm{Sq}^{4 v}=D\left(\mathrm{Sq}^{2 u} \mathrm{Sq}^{2 v}\right)+E\left(\mathrm{Sq}^{u} \mathrm{Sq}^{v}\right)
$$

In particular for any $k \geqq 1$,

$$
\left(\mathrm{Sq}^{2^{k+1}}\right)^{2}=D\left(\left(\mathrm{Sq}^{2^{k}}\right)^{2}\right)+E\left(\left(\mathrm{Sq}^{2^{k-1}}\right)^{2}\right)
$$

Therefore, if $L_{k}$ is the number of Milnor basic operations in $\left(\mathrm{Sq}^{2^{k}}\right)^{2}, k \geqq 0, L_{k+1}=$ $L_{k}+L_{k-1}$. That is, $\left\{L_{k}\right\}_{k \geqq 0}$ is the Fibonacci sequence $\{0,1,1,2,3,5,8,13,21, \ldots\}$.

Proof. By Theorem 6.2(1) and (4), $\mathrm{Sq}^{4 u} \mathrm{Sq}^{4 v}=D\left(\mathrm{Sq}^{2 u} \mathrm{Sq}^{2 v}\right)+B\left(\mathrm{Sq}^{u-2} \mathrm{Sq}^{v-1}\right)$, where $B=A_{1} A_{2} D A_{1} A_{2} D: \mathrm{Sq}^{t_{1, t_{2}}} \mapsto \mathrm{Sq}^{4 t_{1}+3,4 t_{2}+3}$. The identity

$$
F=\frac{1}{1+(\alpha+\beta) \lambda_{1}}+\left(\alpha^{2} \beta \lambda_{2}\right) \cdot F
$$

shows that

$$
\sum_{r, s} f_{r, s}\left(\lambda_{1}, \lambda_{2}\right) \alpha^{r} \beta^{s}=\frac{1}{1+(\alpha+\beta) \lambda_{1}}+\sum_{r, s} f_{r, s}\left(\lambda_{1}, \lambda_{2}\right) \lambda_{2} \alpha^{r+2} \beta^{s+1}
$$

Replacing $\lambda_{i}$ with $\lambda_{i}^{4}$ and multiplying with $\lambda_{1}^{3} \lambda_{2}^{-1}$, we get

$$
\sum_{r, s} f_{r, s}\left(\lambda_{1}^{4}, \lambda_{2}^{4}\right) \lambda_{1}^{3} \lambda_{2}^{-1} \alpha^{r} \beta^{s}=\frac{\lambda_{2}^{-1}}{1+(\alpha+\beta) \lambda_{1}^{4}}+\sum_{r, s} f_{r, s}\left(\lambda_{1}^{4}, \lambda_{2}^{4}\right) \lambda_{1}^{3} \lambda_{2}^{3} \alpha^{r+2} \beta^{s+1}
$$

Since any negative power of $\lambda_{i}$ corresponds to a zero operation in the Steenrod algebra, the coefficient of $\alpha^{u} \beta^{v}$ implies that $E\left(\mathrm{Sq}^{u} \mathrm{Sq}^{v}\right)=B\left(\mathrm{Sq}^{u-2} \mathrm{Sq}^{v-1}\right)$. We have proved the formula.

The formulas obtained can be well derived from an argument of the Milnor matrix 
calculation 2.3. But the technique of formal power series benefits us when we generalize Theorem 6.2(1) and (2)

\section{Notation 6.4.}

$$
\begin{aligned}
\mathrm{Sq}\langle n+1, \xi\rangle & =\sum_{r_{1}, \ldots, r_{n}, r_{n+1}} \mathrm{Sq}^{2 r_{1}+1, \ldots, 2 r_{n}+1,2 r_{n+1}} \xi_{1}^{r_{1}} \ldots \xi_{n}^{r_{n}} \xi_{n+1}^{r_{n+1}} \\
\mathrm{Sq}[n, \xi] & =\sum_{r_{1}, \ldots, r_{n-1}, r_{n}} \mathrm{Sq}^{2 r_{1}+1, \ldots, 2 r_{n-1}+1,2 r_{n}+1} \xi_{1}^{r_{1}} \ldots \xi_{n-1}^{r_{n-1}} \xi_{n}^{r_{n}+1} \\
\mathrm{Sq}[\alpha] & =\sum_{r} \mathrm{Sq}^{2 r+1} \alpha^{r+1} .
\end{aligned}
$$

Theorem 6.5. Suppose that $\xi$ and $\eta$ are sequences of scalar parameters and that the elements $\sigma_{1}, \sigma_{2}, \ldots, \sigma_{n}$ are the 2-weighted symmetric polynomials for the parameters $\alpha_{1}, \alpha_{2}, \ldots, \alpha_{n}$. Then

(1) $\mathrm{Sq}[n, \xi] \cdot \mathrm{Sq}\langle m+1, \eta\rangle=\mathrm{Sq}\langle n+m+1, \xi * \eta\rangle \cdot \xi_{n}^{2^{m}}$,

(2) $\mathrm{Sq}[n, \xi] \cdot \mathrm{Sq}[m, \eta]=\mathrm{Sq}[n+m, \xi * \eta]$,

(3) $\mathrm{Sq}\left[\alpha_{1}\right] \mathrm{Sq}\left[\alpha_{2}\right] \ldots \mathrm{Sq}\left[\alpha_{n}\right]=\mathrm{Sq}[n, \sigma]$.

Proof. The statement (2) can be obtained from the statement (1) by setting $\eta_{m+1}=0$. Iteration of (2) implies the third statement. We prove the first.

Let $W$ be the $\mathbb{F}_{2}$-space generated by all monomials $\xi_{1}^{r_{1}} \ldots \xi_{n}^{r_{n}} \eta_{1}^{s_{1}} \ldots \eta_{m}^{s_{m}} \eta_{m+1}^{s_{m+1}}$ such that either at least one of the numbers $r_{1}, \ldots, r_{n}, s_{1}, \ldots, s_{m}$ is even or $s_{m+1}$ is odd. By $X \equiv Y$ we mean $X-Y \in W$. Consider the formal power series

$$
F=\prod_{k=1}^{n+m+1} \frac{1}{\left(1-\Omega_{k} \lambda_{k}\right)}, \quad \text { over } \mathbb{F}_{2},
$$

where $\Omega$ is the star operation of $\xi$ and $\eta$ :

$$
\begin{array}{ll}
\Omega_{1} & =\xi_{1}+\eta_{1} \\
\Omega_{2} & =\xi_{2}+\xi_{1}^{2} \eta_{1}+\eta_{2} \\
& \vdots \\
\Omega_{n} & =\xi_{n}+\xi_{n-1}^{2} \eta_{1}+\cdots+\eta_{n} \\
\Omega_{n+1} & =\xi_{n}^{2} \eta_{1}+\xi_{n-1}^{2} \eta_{2}+\cdots+\eta_{n+1} \\
& \vdots \\
\Omega_{n+m} & =\xi_{n}^{2^{m}} \eta_{m}+\xi_{n-1}^{2^{m+1}} \eta_{m+1} \\
\Omega_{n+m+1} & =\xi_{n}^{2^{m+1}} \eta_{m+1} .
\end{array}
$$


We observe that for every $\xi_{i}$ has even powers in $\Omega_{j}$ for $j \geqq i+1$, and that every $\eta_{i}$ does not appear in $\Omega_{n+j}$ for $j \geqq i+1$. This implies the congruence

$$
\begin{aligned}
\prod_{k=1}^{n+m+1}\left(1-\Omega_{k} \lambda_{k}\right) & \equiv \xi_{1} \lambda_{1} \cdot \prod_{k=2}^{n+m+1}\left(1-\Omega_{k} \lambda_{k}\right) \\
& \equiv \xi_{1} \lambda_{1} \cdot \xi_{2} \lambda_{2} \cdot \prod_{k=3}^{n+m+1}\left(1-\Omega_{k} \lambda_{k}\right) \\
& \vdots \\
& \equiv \xi_{1} \lambda_{1} \ldots \xi_{n} \lambda_{n} \cdot \prod_{k=n+1}^{n+m+1}\left(1-\Omega_{k} \lambda_{k}\right) \\
& \equiv \xi_{1} \lambda_{1} \ldots \xi_{n} \lambda_{n} \cdot \xi_{n}^{2} \eta_{1} \lambda_{n+1} \prod_{k=n+2}^{n+m+1}\left(1-\Omega_{k} \lambda_{k}\right) \\
& \vdots \\
& \equiv \xi_{1} \lambda_{1} \ldots \xi_{n} \lambda_{n} \cdot \xi_{n}^{2} \eta_{1} \lambda_{n+1} \ldots \xi_{n}^{2 m} \eta_{m} \lambda_{n+m} \cdot\left(1-\Omega_{n+m+1} \lambda_{n+m+1}\right) \\
& \equiv \xi_{1} \lambda_{1} \ldots \xi_{n} \lambda_{n} \cdot \xi_{n}^{2} \eta_{1} \lambda_{n+1} \ldots \xi_{n}^{2 m} \eta_{m} \lambda_{n+m} .
\end{aligned}
$$

Let $F=\sum_{R, S} f_{R, S}(\lambda) \xi^{R} \eta^{S}$. Then $F^{2}=\sum_{R, S} f_{R, S}\left(\lambda^{2}\right) \xi^{2 R} \eta^{2 S}$, where $\lambda^{2}=\left(\lambda_{1}^{2}, \lambda_{2}^{2}, \ldots\right)$. Let

$$
I=(\underbrace{1, \ldots, 1}_{n}, 0, \ldots) \text { and } J=(\underbrace{1, \ldots, 1}_{m}, 0,0, \ldots) \text {. }
$$

Since $F=\prod_{k=1}^{n+m+1}\left(1-\Omega_{k} \lambda_{k}\right) \cdot F^{2}$, modulo $W$,

$$
\sum_{U, V} f_{2 U+I, 2 V+J}(\lambda) \xi^{2 U+I} \eta^{2 V+J}=\sum_{R, S} f_{R, S}\left(\lambda^{2}\right) \xi^{2 R} \eta^{2 S} \cdot \xi_{1} \lambda_{1} \ldots \xi_{n} \lambda_{n} \cdot \xi_{n}^{2} \eta_{1} \lambda_{n+1} \ldots \xi_{n}^{2 m} \eta_{m} \lambda_{n+m}
$$

This is actually an identity for all terms on both sides are not in $W$. After cancelling the factor $\xi_{1} \ldots \xi_{n} \eta_{1} \ldots \eta_{m}$ from both sides and replacing $\xi_{i}^{2}$ with $\xi_{i}$ and $\eta_{j}^{2}$ with $\eta_{j}$, we convert the identity into a formula of Steenrod operations. We find that this is exactly (1).

\section{Nilpotence}

We discuss consequences of Theorem 6.5 in the study of nilpotence in the Steenrod algebra. Recall that an operation $\theta$ in $\mathscr{A}(p)$ has nilpotence $n=: \operatorname{Nil}(\theta)$ if $\theta^{n}=0$ and $\theta^{n-1} \neq 0$. J. Milnor [7] showed that every Steenrod operation of positive degree has a finite nilpotence. He raised the question of calculating the nilpotence in the Steenrod algebra. In 1975, S. W. Wilson conjectured that $\mathrm{Nil}\left(\mathrm{Sq}^{2^{k}}\right)=2 k+2$. D. Davis announced [4] the first results that $\mathrm{Nil}\left(\mathrm{Sq}^{2 k}\right) \geqq 2 k+2$ for $k \geqq 1$ and that Wilson conjecture is true up to $k=5$. K. G. Monks $[9,10]$ extended his results to the operations $\mathscr{P}_{\ell}^{2 *}$ and conjectured that $\operatorname{Nil}\left(\mathscr{P}_{\ell}^{22}\right)=2[s / \ell]+2$. On the other hand, he initialized the study of determining the 
nilpotence of operations $\mathrm{Sq}^{2 r+1}$. He introduced a subalgebra $\mathcal{O}$ of the Steenrod algebra $\mathscr{A}(2)$, containing all operations of the form $\mathrm{Sq}^{2 r+1}$. By an elaborated analysis of duality and matrix he proved a number of theorems. In this section we show that Monks results can be obtained and extended more systematically from our Theorem 6.5 . The subalgebra $\mathcal{O}$ has Adem relations, indicating that the study of nilpotence in $\mathcal{O}$ has the same difficulty as in $\mathscr{A}(2)$. In particular we show that to prove the Wilson conjecture is to prove a relation of operations in $\mathcal{O}$.

Theorem 7.1 ([10]). For any integers $r_{1}, r_{2}, \ldots, r_{n}$,

$$
\operatorname{Nil}\left(\mathrm{Sq}^{2 r_{1}+1,2 r_{2}+1, \ldots, 2 r_{n}+1}\right) \leqq \min \left\{k \mid r_{n}<2^{(k-1) n}-1\right\}
$$

Proof. By iterating Theorem 6.5(2) we have

$$
\mathrm{Sq}\left[n, \xi_{1}\right] \mathrm{Sq}\left[n, \xi_{2}\right] \ldots \mathrm{Sq}\left[n, \xi_{k}\right]=\mathrm{Sq}\left[k n, \xi_{1} * \xi_{2} * \ldots * \xi_{k}\right] \cdot \xi_{1, n}^{2^{(k-1) n}-1} \ldots \xi_{k-1, n}^{2^{n}-1} \text {. }
$$

The coefficient of $\xi_{1,1}^{r_{1}} \ldots \xi_{1, n}^{r_{n}} \ldots \xi_{k, 1}^{r_{1}} \ldots \xi_{k, n}^{r_{n}}$ is the operation $\mathrm{Sq}^{2 r_{1}+1, \ldots, 2 r_{n}+1}$ on the left hand side, and is zero on the right hand side if $r_{n}<2^{(k-1) n}-1$. Therefore the nilpotence of the operation $\mathrm{Sq}^{2 r_{1}+1, \ldots, 2 r_{n}+1}$ is not bigger than the minimal $k$ such that $r_{n}<$ $2^{(k-1) n}-1$.

Theorem 7.2. For any integers $r_{1}, r_{2}, \ldots, r_{n}$,

$$
\mathrm{Sq}^{2 r_{1}+1} \mathrm{Sq}^{2 r_{2}+1} \ldots \mathrm{Sq}^{2 r_{n}+1}=0 \text { if and only if } \mathrm{Sq}^{r_{1}-\left(2^{n-1}-1\right)} \ldots \mathrm{Sq}^{r_{n}-\left(2^{0}-1\right)}=0
$$

As a consequence, the nilpotence of the operation $\mathrm{Sq}^{2 r+1}$ is the minimal positive integer $k$ such that

$$
\mathrm{Sq}^{r-\left(2^{k-1}-1\right)} \ldots \mathrm{Sq}^{r-(2-1)} \mathrm{Sq}^{r}=0
$$

Proof. Let $\sigma_{1}, \sigma_{2}, \ldots, \sigma_{n}$ be the 2-weighted symmetric polynomials for $\alpha_{1}, \alpha_{2}, \ldots, \alpha_{n}$. Suppose $\quad R=\left(r_{1}, \ldots, r_{n}, 0, \ldots\right), \quad T=\left(t_{1}, \ldots, t_{n}, 0, \ldots\right), \quad I=(1, \ldots, 1,0, \ldots) \quad$ and $\quad J=$ $\left(2^{n-1}, \ldots, 2^{0}, 0, \ldots\right)$. From Theorem 6.5(3).

$$
\mathrm{Sq}^{2 r_{1}+1} \mathrm{Sq}^{2 r_{2}+1} \ldots \mathrm{Sq}^{2 r_{n}+1}=\sum_{T}\left[\begin{array}{c}
\sigma^{T+\Delta_{n}} \\
\alpha^{R+I}
\end{array}\right] \mathrm{Sq}^{2 T+I}=\sum_{T}\left[\begin{array}{c}
\sigma^{T} \\
\alpha^{R-J+I}
\end{array}\right] \mathrm{Sq}^{2 T+I}
$$

which is zero if and only if

$$
0=\sum_{T}\left[\begin{array}{c}
\sigma^{T} \\
\alpha^{R-J+1}
\end{array}\right] \mathrm{Sq}^{T}=\mathrm{Sq}^{r_{1}-\left(2^{n-1}-1\right)} \mathrm{Sq}^{r_{2}-\left(2^{n-2}-1\right)} \ldots \mathrm{Sq}^{r_{n}-\left(2^{0}-1\right)}, \text { by Theorem 5.2 }
$$


Theorem 7.3 ([10]). Let $\mathcal{O}$ be the $\mathbb{F}_{2}$-subspace of $\mathscr{A}(2)$ generated by all Steenrod squares of the form $\mathrm{Sq}^{2 r_{1}+1,2 r_{2}+1, \ldots, 2 r_{n}+1}, n \geqq 0$. Then

(1) $\mathcal{O}$ is a subalgebra of $\mathscr{A}(2)$.

(2) The Adem relations in $\mathcal{O}$ are

$$
\mathrm{Sq}^{2 a+1} \mathrm{Sq}^{2 b+1}=\sum_{j=0}^{[(a-1) / 2]}\left[\begin{array}{c}
b-j-1 \\
a-2 j-1
\end{array}\right] \mathrm{Sq}^{2(a+b-j)+1} \mathrm{Sq}^{2 j+1}, \quad a, b \geqq 0 .
$$

(3) The map

$$
\begin{cases}\lambda: \mathcal{O} & \rightarrow \mathcal{O} \\ \mathrm{Sq}^{2 r_{1}+1,2 r_{2}+1, \ldots, 2 r_{n}+1} & \mapsto \mathrm{Sq}^{4 r_{1}+3, \ldots, 4 r_{n-1}+3,4 r_{n}+1}\end{cases}
$$

is an algebraic monomorphism.

Proof. As we see in Theorem 6.5(2) any product of elements from $\mathcal{O}$ is a sum in $\mathcal{O}$. Hence $\mathcal{O}$ is a subalgebra of $\mathscr{A}(2)$. The Bullet-Macdonald technique of residue computation is applied to the case $n=2$ of Theorem 6.5(3). The Adem relations (2) follow. To prove (3), iterate the process of taking modulo $W$ in the proof of Theorem 6.5. We then have

$$
\begin{aligned}
& \sum \mathrm{Sq}^{4 r_{1}+3, \ldots .4 r_{n-1}+3,4 r_{n}+1} \mathrm{Sq}^{4 s_{1}+3, \ldots, 4 s_{m-1}+3,4 s_{m}+1} \xi_{1}^{r_{1}} \ldots \xi_{n-1}^{r_{n}} \xi_{n}^{r_{n}+1} \eta_{1}^{s_{1}} \ldots \eta_{m-1}^{s_{m-1}} \eta_{m}^{s_{m}+1} \\
& =\sum \mathrm{Sq}^{4 t_{1}+3, \ldots .44 t_{n+m-1}+3,4 r_{n+m}+1}(\xi * \eta)_{1}^{t_{1}} \ldots(\xi * \eta)_{n+m-1}^{s_{n+m-1}}(\xi * \eta)_{n+m}^{n_{n+m}+1} .
\end{aligned}
$$

We see that the product rule for operations $\mathrm{Sq}^{4 r_{1}+3, \ldots, 4 r_{n-1}+3,4 r_{n}+1}$ is exactly the same as the one for operations $\mathrm{Sq}^{2 r_{1}+1, \ldots, 2 r_{n-1}+1,2 n+1}$. Hence the $\lambda$-map is algebraic. It is also monic. We have completed the proof.

$\mathcal{O}$ is a "small" subalgebra of $\mathscr{A}(2)$ in the sense that it has less dimension in every degree. But it is equally complicated as $\mathscr{A}(2)$, for both algebra structures are determined by the same star operation. Every formula in $\mathscr{A}(2)$ has a corresponding one in $\mathcal{O}$; the problem of determining nilpotence in $\mathcal{O}$ is the same difficulty as the problem of determining nilpotence in $\mathscr{A}(2)$. Taking another point of view, we can define a different algebra structure in the $\mathbb{F}_{2}$-space $\mathscr{A}(2)$ in which the product of any two basic operations has fewer non-zero terms.

Theorem 7.4. Let $\mathscr{U}$ be $\mathscr{A}(2)$ as an $\mathbb{F}_{2}$-space. Define a product $\dot{x}$ in $\mathscr{U}$, called the reduced product $\hat{x}$ of Steenrod operations, by

$$
\mathrm{Sq}^{r_{1}, \ldots, r_{m}-1, r_{n}+1} \hat{\times} \mathrm{Sq}^{s_{1}, \ldots, s_{m-1}, s_{m}+1}=\sum \mathrm{Sq}^{i_{1}, \ldots, t_{n+m-1}, t_{n+m}+1}, \quad n, m \geqq 0,
$$

where $\mathrm{Sq}^{t_{1} \ldots \ldots t_{n+m-1}, t_{n+m}+1}$ is a non-zero summand of $\mathrm{Sq}^{r_{1} \ldots \ldots, r_{n-1}, r_{n}+1} \cdot \mathrm{Sq}^{s_{1} \ldots \ldots s_{m-1}, s_{m}+1}$. Let 


$$
\mathrm{Sq}^{(0)}(\xi)=1, \quad \mathrm{Sq}^{(n)}(\xi)=\sum \mathrm{Sq}^{r_{1} \ldots, r_{n-1}, r_{n}+1} \xi_{1}^{r_{1}} \ldots \xi_{n-1}^{r_{n-1} \xi_{n}^{r_{n}+1}}, \quad n \geqq 1
$$

Then

(1) $\mathscr{U}$ is an algebra, and for $n, m \geqq 0$

$$
\mathrm{Sq}^{(n)}(\xi) \mathrm{Sq}^{(m)}(\eta)=\mathrm{Sq}^{(n+m)}(\xi * \eta)
$$

(2) $\mathscr{U}$ is algebraically isomorphic to $\mathcal{O}$ under the map $\mathrm{Sq}^{r_{1}, \ldots, r_{n-1}, r_{n}+1} \mapsto$

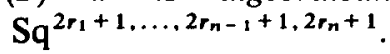

(3) If $A, B \in \mathcal{O}$, then $A \dot{\times} B=A \cdot B \in \mathcal{O}$.

(4) The Adem relations are

$$
\mathrm{Sq}^{a+1} \hat{\times} \mathrm{Sq}^{b+1}=\sum_{j=0}^{[(a-1) / 2]}\left[\begin{array}{c}
b-j-1 \\
a-2 j-1
\end{array}\right] \mathrm{Sq}^{a+b-j+1} \hat{\times} \mathrm{Sq}^{j+1}, \quad a, b \geqq 0 .
$$

Proof. (1). Let $R=\left(r_{1}, \ldots, r_{n}, 0, \ldots\right), S=\left(s_{1}, \ldots, s_{m}, 0 \ldots\right)$ and $T=\left(t_{1}, \ldots, t_{n+m}, 0, \ldots\right)$. By Theorem 3.5,

$$
\begin{gathered}
\mathrm{Sq}^{R+\Delta_{n}} \hat{\times} \mathrm{Sq}^{S+\Delta_{m}}=\sum_{t_{n+m} \neq 0}\left[\begin{array}{c}
(\xi * \eta)^{T} \\
\xi^{R+\Delta_{n}} \eta_{m}^{S+\Delta_{m}}
\end{array}\right] \mathrm{Sq}^{T}=\sum_{T}\left[\begin{array}{c}
(\xi * \eta)^{T} \\
\xi^{R+\Delta_{n}} \eta_{m}^{S+\Delta_{m}}
\end{array}\right] \mathrm{Sq}^{T+\Delta_{n+m}} \\
\sum_{R, S} \mathrm{Sq}^{R+\Delta_{n}} \hat{\times} \mathrm{Sq}^{S+\Delta_{m}} \xi^{R+\Delta_{n}} \eta^{S+\Delta_{m}}=\sum_{T} \mathrm{Sq}^{T+\Delta_{n+m}}(\xi * \eta)^{T+\Delta_{n+m}}
\end{gathered}
$$

That is, $\mathrm{Sq}^{(n)}(\xi) \hat{\times} \mathrm{Sq}^{(m)}(\eta)=\mathrm{Sq}^{(n+m)}(\xi * \eta)$. Since the star operation is associative so is the reduced product $\hat{x}$. Hence $\mathscr{U}$ is an algebra. (2). The map defined maps the formula in (1) to the formula in Theorem 6.5(2). Since it is both monic and epic, it is isomorphic. (3). We note from Theorem 6.5(2) that if $\mathrm{Sq}^{t_{1}, t_{2}, \ldots, t_{n+m}}$ is a non-zero summand of $\mathrm{Sq}^{r_{1}, r_{2} \ldots, \ldots, r_{n}} \cdot \mathrm{Sq}^{s_{1}, s_{2}, \ldots, s_{m}}$, where all $r_{i}$ and $s_{j}$ are odd, then $t_{m+m}$ is odd. (4). The Adem relations can be obtained from those for the algebra $\mathcal{O}$ via the map in (2).

Theorem 7.5. For any integers $r_{1}, r_{2}, \ldots, r_{n}$, and $k \geqq 1$

(1) $\mathrm{Sq}^{2 r_{1}+1} \mathrm{Sq}^{2 r_{2}+1} \ldots \mathrm{Sq}^{2 r_{n}+1}=0$ if and only if $\mathrm{Sq}^{r_{1}+1} \hat{\times} \mathrm{Sq}^{r_{2}+1} \hat{\times} \cdots \hat{\times} \mathrm{Sq}^{r_{n}+1}=0$,

(2) $\mathrm{Sq}^{r_{1}} \mathrm{Sq}^{r_{2}} \ldots \mathrm{Sq}^{r_{n}}=0$ if and only if $\mathrm{Sq}^{r_{1}+2^{n-1}-1} \hat{\times} \mathrm{Sq}^{r_{2}+2^{n-2}-1} \hat{x} \cdots \hat{\times} \mathrm{Sq}^{r_{n}+2^{0-1}}=0$,

(3) $\left(\mathrm{Sq}^{2^{k-1}}\right)^{2 k}=0$ if and only if $\mathrm{Sq}^{2^{k+1}+2^{2 k-1}} \cdot \mathrm{Sq}^{2^{k+1}+2^{2 k-1}-1} \ldots \mathrm{Sq}^{2^{k+1}+21-1}=0$.

Proof. Let $\sigma_{i}$ be the 2-weighted symmetric polynomials for the $\alpha_{i}$. Let $R=$ $\left(r_{1}, \ldots, r_{n}, 0, \ldots\right), T=\left(t_{1}, \ldots, t_{n}, 0, \ldots\right), I=(1, \ldots, 1,0, \ldots)$ and $J=\left(2^{n-1}, \ldots, 2^{0}, 0, \ldots\right)$. By Theorem 6.5(3)

$$
\mathrm{Sq}^{2 r_{1}+1} \mathrm{Sq}^{2 r_{2}+1} \ldots \mathrm{Sq}^{2 r_{n}+1}=\sum_{T}\left[\begin{array}{c}
\sigma^{T+\Delta_{n}} \\
\alpha^{R+1}
\end{array}\right] \mathrm{Sq}^{2 T+I}
$$


which is zero if and only if

$$
\sum_{T}\left[\begin{array}{c}
\sigma^{T+\Delta_{n}} \\
\alpha^{R+I}
\end{array}\right] \mathrm{Sq}^{T+\Delta_{n}}=0
$$

The left hand side is equal to $\mathrm{Sq}^{r_{1}+1} \hat{x} \mathrm{Sq}^{r_{2}+1} \hat{x} \cdots \hat{x} \mathrm{Sq}^{r_{n}+1}$. Likewise, by Theorem 5.2 in the case $\ell=1$,

$$
\mathrm{Sq}^{r_{1}} \ldots \mathrm{Sq}^{r_{n}}=\sum_{T}\left[\begin{array}{c}
\sigma^{T} \\
\alpha^{R}
\end{array}\right] \mathrm{Sq}^{T}=\sum_{T}\left[\begin{array}{c}
\sigma^{T+\Delta_{n}} \\
\alpha^{R+J}
\end{array}\right] \mathrm{Sq}^{T}
$$

which is zero if and only if

$$
0=\sum_{T}\left[\begin{array}{c}
\sigma^{T+\Delta_{n}} \\
\alpha^{R+J}
\end{array}\right] \mathrm{Sq}^{T+\Delta_{n}}=\mathrm{Sq}^{r_{1}+2^{n-1}} \hat{\times} \cdots \hat{\times} \mathrm{Sq}^{r_{n-1}+2} \hat{\times} \mathrm{Sq}^{r_{n}+1}, \quad \text { by Theorem } 7.4
$$

The formula (3) is implied by the joint of (1) and (2).

Theorem $7.6([10])$. For an integer $r$

$$
\max \left\{k \mid r \equiv-1\left(\bmod 2^{k-1}\right)\right\}<\operatorname{Nil}\left(\mathrm{Sq}^{2 r+1}\right) \leqq \min \left\{k \mid r<2^{k-1}-1\right\} .
$$

Proof. The upper bound is given by Theorem 7.1. The lower bound is a consequence of Theorem 7.5(1) and the assertion that for any $k$ such that $2^{k}$ divides $r+1$, $\mathrm{Sq}^{(r+1) / 2,(r+1) / 2^{2} \ldots \ldots(r+1) / 2^{k},(r+1) / 2^{k}}$ is a non-zero term in

$$
\underbrace{\mathrm{Sq}^{r+1} \ldots \mathrm{Sq}^{r+1}}_{n} \text {, hence a non zero term in } \underbrace{\mathrm{Sq}^{r+1} \hat{x} \cdots \hat{\times} \mathrm{Sq}^{r+1}}_{n} \text {. }
$$

This is checked either by the Milnor matrix calculation $2.3(1)$ or by using weighted symmetric polynomials. We omit the details.

\section{Dickson invariants and Mui invariants}

Definition 8.1. Given a set of elements $x_{1}, x_{2}, \ldots, x_{n}$ the Dickson invariants $c_{n, n-1}, c_{n, n-2}, \ldots, c_{n, 0}$ and the Mui invariants $\phi_{n-1}, \phi_{n-2}, \ldots, \phi_{0}$ and the modified $M u i$ invariants $\bar{\phi}_{n-1}, \tilde{\phi}_{n-2}, \ldots, \tilde{\phi}_{0}$ over the field $\mathbb{F}_{q}$ are defined respectively by

$$
\begin{gathered}
\prod_{a_{1}, \ldots, a_{n} \in \mathrm{F}_{q}}\left(a_{1} x_{1}+\cdots+a_{n} x_{n}+X\right)=X^{q^{n}}-c_{n, n-1} X^{q^{n-1}}+\cdots+(-1)^{n} c_{n, 0} X, \\
\phi_{j}=\prod_{a_{1}, \ldots, a_{j \in \mathrm{F}_{q}}}\left(a_{1} x_{1}+\cdots+a_{j} x_{j}+x_{j+1}\right),
\end{gathered}
$$




$$
\bar{\phi}_{j}=\prod_{a_{1}, \ldots, a_{j}, a_{j+1} \in \mathbb{F}_{q}, a_{j+1} \neq 0}\left(a_{1} x_{1}+\cdots+a_{j} x_{j}+a_{j+1} x_{j+1}\right)=-\phi_{j}^{q-1}
$$

In the case that $\left\{x_{1}, x_{2}, \ldots, x_{n}\right\}$ is algebraically independent over $\mathbb{F}_{q}$, they are indeed invariants of linear transformations. Let us make this precise.

Let $\mathrm{GL}_{n}(q)$ be the group of all non-singular $n \times n$-matrices over $\mathbb{F}_{q}, \tilde{T}_{n}(q)$ be the subgroup of $\mathrm{GL}_{n}(q)$ consisting of triangular matrices; $\mathrm{T}_{n}(q)$ be the subgroup of $\tilde{\mathrm{T}}_{n}(q)$ consisting of matrices in which all diagonal elements are 1 . Recall that $T_{n}(q)$ is a Sylow subgroup of $\mathrm{GL}_{n}(q)$ and $\widetilde{\mathrm{T}}_{n}(q)$ is the normalizer of $\mathrm{T}_{n}(q)$. Suppose that $x_{1}, x_{2}, \ldots, n_{n}$ is $\mathbb{F}_{q}$-linearly independent. There is an action of the groups $\operatorname{GL}_{n}(q), \mathrm{T}_{n}(q)$ and $\widetilde{T}_{n}(q)$ on the symmetric algebra $\mathbb{F}_{q}\left[x_{1}, x_{2}, \ldots, x_{n}\right]$ generated by the vector space $V=\mathbb{F}_{q}\left(x_{1}, x_{2}, \ldots, x_{n}\right)$, which algebraically extends the linear action on $V$ :

$$
A \cdot x=\left(x_{1}, \ldots, x_{n}\right) \cdot A\left(\begin{array}{c}
a_{1} \\
\vdots \\
a_{n}
\end{array}\right) \begin{aligned}
& \text { where } A \in \mathrm{GL}_{n}(q) \\
& x=a_{1} x_{1}+a_{2} x_{2}+\cdots+a_{n} x_{n} .
\end{aligned}
$$

An element $f \in \mathbb{F}_{q}\left[x_{1}, x_{2}, \ldots, x_{n}\right]$ is said an invariant of the group $G\left(\mathrm{GL}_{n}(q), \mathrm{T}_{n}(q)\right.$ or $\left.\widetilde{\mathrm{T}}_{n}(q)\right)$ if $A \cdot f=f$ for any $A \in G$. Clearly all invariants form a subalgebra $\mathbb{F}_{q}\left[x_{1}, x_{2}, \ldots, x_{n}\right]^{G} \subset \mathbb{F}_{q}\left[x_{1}, x_{2}, \ldots, x_{n}\right]$. For the three groups $\operatorname{GL}_{n}(q), \mathrm{T}_{n}(q)$ and $\tilde{\mathrm{T}}_{n}(q)$ they are actually polynomial algebras of Krull dimension $n$.

\section{Theorem 8.2.}

(1) ([5]) $\mathbb{F}_{q}\left[x_{1}, x_{2}, \ldots, x_{n}\right] \mathrm{GL}_{n}(q)=\mathbb{F}_{q}\left[c_{n, 0}, c_{n, 1}, \ldots, c_{n, n-1}\right]$,

(2) ([11]) $\mathbb{F}_{q}\left[x_{1}, x_{2}, \ldots, x_{n}\right] \mathbb{T}_{n}(q)=\mathbb{F}_{q}\left[\phi_{0}, \phi_{1}, \ldots, \phi_{n-1}\right]$,

$$
\mathbb{F}_{q}\left[x_{1}, x_{2}, \ldots, x_{n}\right] \tilde{\mathrm{T}}_{n}(q)=\mathbb{F}_{q}\left[\tilde{\phi}_{0}, \tilde{\phi}_{1}, \ldots, \tilde{\phi}_{n-1}\right]
$$

Proof. The statements (1) and (2) in the case $q=p$ were proved in [14] and [11]. The same proofs apply to the general case. We need to prove (3). It is not hard to see that $\tilde{\phi}_{i}$ are invariant under the action of $\tilde{T}_{n}(q)$. Now suppose that $f \in \mathbb{F}_{q}\left[x_{1}, x_{2}, \ldots, x_{n}\right]$ is invariant of $\tilde{\mathrm{T}}_{n}(q)$. It must be an invariant of $\mathrm{T}_{n}(q)$ for $\mathrm{T}_{n}(q) \subset \tilde{\mathrm{T}}_{n}(q)$. Therefore it is a polynomial in the elements $\phi_{i}$ by (2). For a fixed $i$ let $f=\sum_{k \geqq 0} f_{k} \phi_{i}^{k}$, where each $f_{k}$ is a polynomial in $\left\{\phi_{j}\right\}_{j \neq i}$. Let $A_{i}(a)$ be the diagonal matrix which has the diagonal elements $(1, \ldots, 1, a, 1, \ldots, 1)$. We verify that

$$
A_{i}(a) \cdot \phi_{j}= \begin{cases}\phi_{j} & \text { if } j \neq i \\ a \phi_{j} & \text { if } j=i\end{cases}
$$

Applying $A_{i}(a)$ to $f$, we have

$$
\sum_{k \geqq 0} f_{k} a^{k} \phi_{i}^{k}=\sum_{k \geqq 0} f_{k} \phi_{i}^{k}
$$


The coefficients on both sides are agreed: $f_{k} \cdot\left(a^{k}-1\right)=0$ for all $k$. If $f_{k} \neq 0$ then for any $0 \neq a \in \mathbb{F}_{q}, a^{k}-1=0 ; k$ is a multiple of $(q-1)$. Hence $f$ is a polynomial in $\mathbb{F}_{q}\left[\tilde{\phi}_{0}, \hat{\phi}_{1}, \ldots, \tilde{\phi}_{n-1}\right]$.

Lemma 8.3. The first Dickson invariants are

$$
c_{1,0}=x_{1}^{q-1}, \quad c_{2,0}=x_{1}^{q-1} x_{2}^{q-1}\left(x_{1}^{q-1}-x_{2}^{q-1}\right)^{q-1}, \quad c_{2,1}=\frac{x_{2}^{q^{2}-1}-x_{1}^{q^{2}-1}}{x_{2}^{q-1}-x_{1}^{q-1}} .
$$

Proof. A routine calculation shows that $\prod_{a_{1} \in F_{q}}\left(a_{1} x_{1}+X\right)=X^{q}-x_{1}^{q-1} X$, and

$$
\begin{aligned}
\prod_{a_{1}, a_{2} \in \mathbf{F}_{q}}\left(a_{1} x_{1}+a_{2} x_{2}+X\right)= & \prod_{a_{2} \in \mathbf{F}_{q}}\left(\prod_{a_{1} \in F_{q}}\left(a_{1} x_{1}+\left(a_{2} x_{2}+X\right)\right)\right) \\
= & \prod_{a_{2} \in \mathbf{F}_{q}}\left(\left(a_{2} x_{2}+X\right)^{q}-x_{1}^{q-1}\left(a_{2} x_{2}+X\right)\right) \\
= & \prod_{a_{2} \in F_{q}}\left(a_{2}\left(x_{2}^{q}-x_{1}^{q-1} x_{2}\right)+\left(X^{q}-x_{1}^{q-1} X\right)\right) \\
= & \left(X^{q}-x_{1}^{q-1} X\right)^{q}-\left(x_{2}^{q}-x_{1}^{q-1} x_{2}\right)^{q-1}\left(X^{q}-x_{1}^{q-1} X\right) \\
= & X^{q^{2}}-\left(x_{1}^{q(q-1)}+\left(x_{2}^{q}-x_{1}^{q-1} x_{2}\right)^{q-1}\right) X^{q} \\
& +x_{1}^{q-1}\left(x_{2}^{q}-x_{1}^{q-1} x_{2}\right)^{q-1} X .
\end{aligned}
$$

The coefficients of $X$ and $X^{q}$ in these polynomials are the first Dickson invariants by definition.

Lemma 8.4. Define $\Lambda_{0}=1$ and for $i=1,2, \ldots, n$

$$
\Lambda_{i}=\left|\begin{array}{cccc}
x_{1} & x_{2} & \ldots & x_{i} \\
x_{1}^{q} & x_{2}^{q} & \ldots & x_{i}^{q} \\
\vdots & \vdots & \vdots & \vdots \\
x_{1}^{q^{i-1}} & x_{2}^{q^{i-1}} & \ldots & x_{i}^{q^{i-1}}
\end{array}\right| \text { (over } \mathbb{F}_{q} \text { ). }
$$

Then $\phi_{i}=\Lambda_{i+1} / \Lambda_{i}$ for $i=0,1, \ldots,(n-1)$. In particular $\phi_{0}=x_{1}, \phi_{1}=x_{2}\left(x_{2}^{q-1}-x_{1}^{q-1}\right)$.

Proof. The determinant

$$
\left|\begin{array}{ccccc}
x_{1} & x_{2} & \ldots & x_{i} & X \\
x_{1}^{q} & x_{2}^{q} & \ldots & x_{i}^{q} & X^{q} \\
\vdots & \vdots & \vdots & \vdots & \vdots \\
x_{1}^{q^{q}} & x_{2}^{q} & \ldots & x^{q} & X^{q}
\end{array}\right|
$$


is a polynomial in $X$ which has roots $a_{1} x_{1}+a_{2} x_{2}+\cdots+a_{i} x_{i}$ for any $a_{1}, a_{2}, \ldots, a_{i} \in \mathbb{F}_{q}$ and has the leading coefficient $\Lambda_{i}$. Therefore it is equal to

$$
\Lambda_{i} \cdot \prod_{a_{1}, \ldots, a_{i} \in \mathrm{F}_{q}}\left(a_{1} x_{1}+a_{2} x_{2}+\cdots+a_{i} x_{i}+X\right) .
$$

Take $X=x_{i+1}$. The determinant above is then $\Lambda_{i+1}$. Hence $\Lambda_{i+1}=\Lambda_{i} \phi_{i}$.

Observation 8.5. In Theorem 5.4, $\alpha_{1}=u(v-u)^{q-1}$ and $\alpha_{2}=v^{q}$. Let $u=x_{2}^{q-1}, v=x_{1}^{q-1}$.

$$
\left\{\begin{array}{c}
\alpha_{1}=x_{2}^{q-1}\left(x_{1}^{q-1}-x_{2}^{q-1}\right)^{q-1}=\tilde{\phi}_{1}, \quad \alpha_{2}=x_{1}^{q(q-1)}=\tilde{\phi}_{0}^{q} \\
\sigma_{1}=\alpha_{1}+\alpha_{2}=u(v-u)^{q-1}+v^{q}=\frac{u^{q+1}-v^{q+1}}{u-v}=\frac{x_{2}^{q^{2}-1}-x_{1}^{q^{2}-1}}{x_{2}^{q-1}-x_{1}^{q-1}}=c_{2,1} \\
\sigma_{2}=\alpha_{1}^{q} \alpha_{2}=u(u-v)^{q^{2}-q} u^{q} v^{q}=\left[\left(x_{2}^{q-1}-x_{1}^{q-1}\right)^{q-1} x_{2}^{q-1} x_{1}^{q-1}\right]^{q}=c_{2,0}^{q}
\end{array}\right.
$$

We see that the Dickson and modified Mui invariants are related by weighted symmetric polynomials. It comes as no surprise to us that this relationship is generalized to:

Theorem 8.6. Suppose that $\sigma_{i}$ are the q-weighted symmetric polynomials for $\alpha_{i}$, and that $c_{n, i}$ and $\tilde{\phi}_{i}$ are the Dickson and modified Mui invariants for the elements $x_{1}, x_{2}, \ldots, x_{n}$.

$$
\text { If }\left\{\begin{array} { c } 
{ \alpha _ { 1 } = \tilde { \phi } _ { n - 1 } } \\
{ \alpha _ { 2 } = \tilde { \phi } _ { n - 2 } ^ { q } } \\
{ \vdots } \\
{ \alpha _ { n } = \tilde { \phi } _ { 0 } ^ { q ^ { n - 1 } } , }
\end{array} \quad \text { then } \left\{\begin{array}{c}
\sigma_{1}=c_{n, n-1} \\
\sigma_{2}=c_{n, n-2}^{q} \\
\vdots \\
\sigma_{n}=c_{n, 0}^{q^{n-1}}
\end{array}\right.\right.
$$

Proof. The theorem is trivially true in the case $n=1$ and is true in the case $n=2$ by the Observation 8.5. We prove the general case by induction on $n$. Suppose $n \geqq 2$. Let $y_{i}=x_{i}^{q}-x_{1}^{q-1} x_{i}$ for $i=2,3, \ldots, n$. It is checked that $\left\{y_{2}, y_{3}, \ldots, y_{n}\right\}$ is $\mathbb{F}_{q}$-linear independent. Let $\psi_{j}$ and $d_{j}, j=1,2, \ldots,(n-1)$, be the modified Mui invariants and the Dickson invariants for $y_{2}, y_{3}, \ldots, y_{n}$. Since for any $a_{2}, \ldots, a_{i+1} \in \mathbb{F}_{q}$,

$$
\begin{aligned}
a_{2} y_{2}+\cdots+ & a_{i+1} y_{i+1}=a_{2}\left(x_{2}^{q}-x_{1}^{q-1} x_{2}\right)+\cdots+a_{i}\left(x_{i}^{q}-x_{1}^{q-1} x_{i}\right)+a_{i+1}\left(x_{i+1}^{q}-x_{1}^{q-1} x_{i+1}\right) \\
& =\left(a_{2} x_{2}+\cdots+a_{i} x_{i}+a_{i+1} x_{i+1}\right)^{q}-x_{1}^{q-1}\left(a_{2} x_{2}+\cdots+a_{i} x_{i}+a_{i+1} x_{i+1}\right) \\
& =\prod_{a_{1} \in \mathrm{F}_{q}}\left(a_{1} x_{1}+a_{2} x_{2}+\cdots+a_{i} x_{i}+a_{i+1} x_{i+1}\right), \\
-\tau_{i} & =\prod_{a_{2} \ldots a_{i+1} \in \mathrm{F}_{q}, a_{i+1} \neq 0}\left(a_{2} y_{2}+\cdots+a_{i} y_{i}+a_{i+1} y_{i+1}\right) \\
& =\prod_{a_{1}, a_{2} \ldots \ldots a_{i}+1 \in \mathrm{F}_{q}, a_{i+1} \neq 0}\left(a_{1} x_{1}+a_{2} x_{2}+\cdots+a_{i} x_{i}+a_{i+1} x_{i+1}\right)=-\tilde{\phi}_{i+1} .
\end{aligned}
$$


Hence $\tilde{\psi}_{i}=\tilde{\phi}_{i+1}$ for $i=1,2, \ldots,(n-1)$. The Dickson invariants for $x_{i}$ and for $y_{j}$ are also related to each other. To show this let

$$
F(X)=X^{q^{n}}-c_{n, n-1} X^{q^{n-1}}+\cdots+(-1)^{n} c_{n, 0} X,
$$

and

$$
G(Y)=Y^{q^{n-1}}-d_{n-2} Y^{q^{n-2}}+\cdots+(-1)^{n-1} d_{0} Y
$$

be the defining polynomials of the Dickson invariants for the elements $x_{1}, x_{2}, \ldots, x_{n}$ and $y_{2}, y_{3}, \ldots, y_{n}$ respectively. Then

$$
\begin{aligned}
G\left(X^{q}-x_{1}^{q-1} X\right)= & X^{q^{n}}-\left(d_{n-2}+x_{1}^{q^{n-1}(q-1)}\right) X^{q^{n-1}} \\
& +\left(d_{n-3}+d_{n-2} x^{q^{n-2}(q-1)}\right) X^{q^{n-2}} \\
& -\cdots+ \\
& +(-1)^{n-1}\left(d_{0}+d_{1} x_{1}^{q(q-1)}\right) X^{q} \\
& +(-1)^{n} d_{0} x_{1}^{q-1} X
\end{aligned}
$$

which is satisfied by $X=x_{i}$ for $i=1,2, \ldots, n$. Hence $F(X)=G\left(X^{q}-x_{1}^{q-1} X\right)$. By comparing the coefficients we obtain

$$
\left\{\begin{aligned}
c_{n, n-1}= & d_{n-2}+x_{1}^{q^{n-1}(q-1)} \\
& \vdots \\
c_{n, 1}= & d_{0}+d_{1} x_{1}^{q(q-1)} \\
c_{n, 0}= & d_{0} x_{1}^{q-1} .
\end{aligned}\right.
$$

Also if $\tau_{1}, \tau_{2}, \ldots, \tau_{n-1}$ are the $q$-weighted symmetric polynomials for $\alpha_{1}, \alpha_{2}, \ldots, \alpha_{n-1}$ then

$$
\sigma_{1}=\tau_{1}+\alpha_{n}, \quad \sigma_{2}=\tau_{2}+\tau_{1}^{q} \alpha_{n}, \ldots, \sigma_{n-1}=\tau_{n-1}+\tau_{n-2}^{q} \alpha_{n}, \quad \sigma_{n}=\tau_{n-1}^{q} \alpha_{n} .
$$

Now by induction $\tau_{1}=d_{n-2}, \tau_{2}=d_{n-3}^{q}, \ldots, \tau_{n-2}=d_{1}^{q^{n-2}}, \tau_{n-1}=d_{g}^{n-1}$. Since $\alpha_{n}=\delta g^{n-1}=$ $x_{1}^{q^{n-1}(q-1)}$ we verify that $\sigma_{1}=c_{n, n-1}, \sigma_{2}=c_{n, n-2}^{q}, \ldots, \sigma_{n-1}=c_{n, 1}^{n^{n-2}}, \sigma_{n}=c_{n, 0}^{q^{n-1}}$. The induction is complete.

Combining Theorem 8.6 and Theorem 5.2 we get

Theorem 8.7.

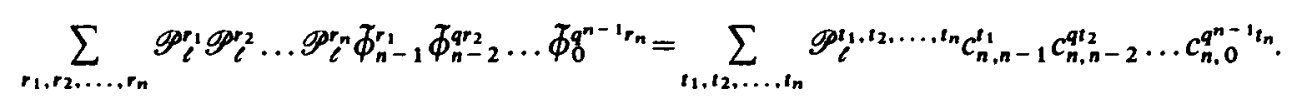

Therefore any iterated product of Steenrod operations is expressed by a sum of Milnor basic operations with coefficients coming from the expansions of monomials of 
Dickson invariants in terms of (modified) Mui invariants. We claim that this is a link of the algebra structure of the Steenrod algebra to the modular representation theory. We can expect a close interaction between these two areas of research. We will come to this topic in a future paper.

\section{REFERENCES}

1. M. Atiyah and F. Hirzebruch, Cohomologie-Operationen und Charakteristische Klassen, Math. Z. 77 (1961), 149-187.

2. S. R. Bullet and I. G. Macdonald, On the Adem relations, Topology, 21 (1982), 329-332.

3. D. Davies, The anti-automorphism of the Steenrod algebra, Proc. Amer. Math. Soc. 44 (1974), 235-236.

4. D. Davies, On the height of $\mathrm{Sq}^{2 i}$, unpublished (1984).

5. L. E. Dickson, A fundamental system of invariants of the general modular linear groups with a solution of the form problem, Trans. Amer. Math. Soc. 12 (1911), 75-98.

6. Z. Li, Formulas for Brown-Peterson operations, Canad. J. Math. 46 (1994), 772-792.

7. J. Milnor, The Steenrod algebra and its dual, Ann. of Math. 67 (1958), 150-171.

8. J. Milnor and J. Moore, On the structure of Hopf algebras, Ann. of Math. 81 (1965), 211-264.

9. K. G. Monks, Nilpotency \& torsion in the Steenrod algebra and its cohomology (Ph.D. Thesis, Lehigh University, 1989).

10. K. G. Monks, Nilpotence in the Steenrod algebra, Preprint (1991).

11. H. Mul, Modular invariant theory and the cohomology algebras of symmetric spaces, $J$. Fac. Sci. Univ. Tokyo 22 (1975), 319-369.

12. F. P. Peterson, Some formulas in the Steenrod algebra, Proc. Amer. Math. Soc. 45 (1974), 291-294.

13. N. E. Steenrod and D. B. A. Epstein, Cohomology operations (Ann. of Math. Studies, 50, Princeton University Press, 1962).

14. C. Wilkerson, A primer of the Dickson invariants, Contemp. Math. 19 (1983), 421-434.

Department of Mathematics

The University of British Columbia

VANCOUVER, B.C., CANADA

V6T 122

Email: zli@math.ubc.ca 